\title{
INMERSIONES EN ESPACIOS TOPOLÓGICOS CONEXOS
}

\author{
Tesis que presenta \\ Andrés Sabino Díaz Castro \\ Para la obtención del grado en la Maestría en \\ Matemáticas
}

Asesor

Richard G. Wilson

Abril de 1999

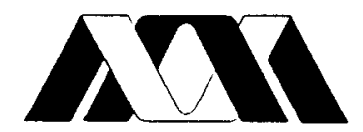

Universidad Autónoma Metropolitana - Iztapalapa División de Ciencias Básicas e Ingeniería 


\section{INMERSIONES EN ESPACIOS \\ TOPOLÓGICOS CONEXOS}

Andrés Sabino Díaz Castro

Maestría en Matemáticas 


\section{Agradecimientos}

Deseo agradecer las observaciones y sugerencias, que hicieron al presente trabajo los profesores:

Dra. Isabel Puga Espinosa de la Facultad de Ciencias de la UnAM.

Dr. Constancio Hernández Garcia

Dr. Mikhail V. Tkačenko

Dr. Valdimir V. Tkachuk

Dr. Richard G. Wilson

Miembros del Departamento de Matemáticas de la Universidad Autónoma Metropolitana, Unidad Iztapalapa.

Al Dr. Richard G. Wilson le agradezco todo su apoyo, que como asesor, me permitió concluir esta tesis.

Este trabajo está dedicado a mis padres y a Norma por el respeto y apoyo a mis decisiones. 


\section{Indice}

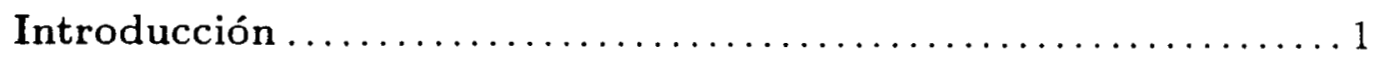

Capítulo 1. Conectificación de espacios $T_{0}, T_{1}$ y de espacios de Hausdorff

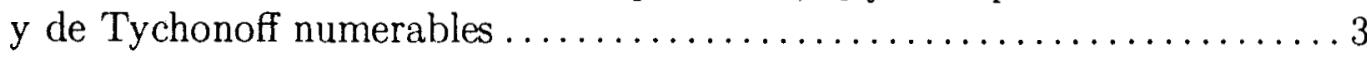

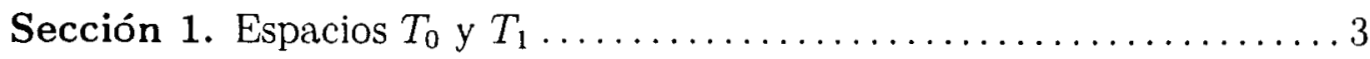

Sección 2. Conectificación de espacios de Hausdorff y de Tychonoff

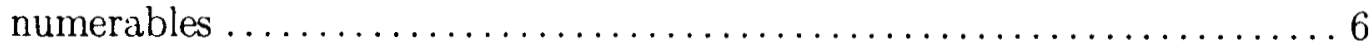

Capitulo 2. Conectificación de espacios de Hausdorff y espacios de Tychonoff............................................ 26

Referencias....................................... 54 


\section{Introducción.}

Se dice que un espacio topológico $X$ es conexo si no existe $U \subset X$ tal que $U \neq \emptyset$ y $U \neq X$ y $c l(U) \cap c l(X \backslash U)=\emptyset$. Esta moderna noción de conexidad fue propuesta por Jordan [1838-1922], en la página 25 de su libro Cours d'Analyse en 1893. Jordan aplicó este concepto para la clase de subconjuntos compactos del plano. La definición para espacios abstractos fue dada por Lennes en 1911 y por Hausdorff [1868-1942] en 1914 quien inició un estudio sistemático de la conexidad.

En 1930, 'Tychonoff dió una caracterización de los espacios que pueden encajarse densamente en un espacio de Hausdorff compacto [18]. Encajes densos en espacios conexos es un tema de estudio relativamente reciente; en [6], se probó que la línea de Sorgenfrey se puede encajar densamente en un espacio de Hausdorff conexo y en [11] se demostró que todo espacio métrico fuertemente zero-dimencional sin subconjuntos abiertos compactos propios, se puede encajar densamente en un espacio ordenado.

En [19], se definió el concepto de un espacio conectificable, como aquel que se puede encajar densamente en un espacio conexo; dicho espacio conexo se llama una conectificación. En [19], se inició un estudio de las hipótesis necesarias y suficientes para la existencia de conectificaciones que satisfacen los mismos axiomas de separación que el espacio mismo y se caracterizaron los espacios que tienen conectificaciones $T_{0}, T_{1}$ y $T_{2}$ numerables. Caracterizaciones para espacios de Hausdorff en general, no son conocidas, sin embargo se sabe que un espacio de Hausdorff conectificable no contiene subconjuntos abicrto-cerrados no vacíos que sean $H$-cerrados.

El presente trabajo es una recopilación de los resultados que aparecen en $[19],[12],[1]$ y [17] y que tratan de la conectificación de los espacios $T_{0}, T_{1}, T_{2}$ y espacios de Hausdorff y de Tychonoff numerables, se incluye también algunos resultados para espacios de Tychonoff en general. El trabajo consta de dos capítulos.

En primer lugar, en el capítulo 1 se caracterizan los espacios que tienen conectificaciónes $T_{0}$ y $T_{1}$ respectivamente y en seguida se trata de espacios de Hausdorff numerables y de espacios de Tychonoff numerables. Se da 
una caracterización para que espacios de Hausdorff numerables tengan una conectificación de Hausdorff. Se define el concepto de $\omega$-conectificabilidad y se dan condiciones suficientes para que espacios de Tychonoff y espacios de Hausdorff numerables sean $\omega$-conectificables.

En el capítulo 2, los espacios considerados son de Hausdorff y de Tychonoff; se establecen condiciones suficientes para que estos espacios tengan conectificaciones de Hausdorff. Se dan dos condiciones suficientes para que espacios metrizables tengan una conectificación de Hausdorff. Se prueba que todo espacio de Hausdorff cuasirregular y denso en sí mismo de cardinalidad menor que el continuo, tiene una conectificación de Hausdorff. Finalmente, se da una caracterización para que un espacio perfectamente normal tenga una conectificación de Hausdorff.

En todo el trabajo, $\omega$ denotará el primer ordinal (cardinal) infinito, $\omega_{1}$ el primer ordinal (cardinal) no numerable y $c$ denotará la cardinalidad del continuo. Si $X$ es un conjunto, $\mathcal{P}(X)$ denotará la familia de todos los subconjuntos de $X$. $\mathbb{R}$ siempre denotará el conjunto de los números reales con la topología de orden. 


\section{CAPÍTULO 1.}

\section{Conectificación de espacios $T_{0}, T_{1}$ y de espacios de Hausdorff y de Tychonoff numerables.}

\section{Sección 1. Espacios $T_{0}$ y $T_{1}$.}

El concepto de conectificar un espacio topológico se introdujo en [19]. Se dice que un espacio topologíco es conectificable si se puede encajarlo densamente en un espacio conexo. En esta sección, se dan condiciones necesarias y suficientes para que un espacio $T_{0}$ tenga una conectificación $T_{0}$ y para que un espacio $T_{1}$ tenga una conectificación $T_{1}$. Se observa que el Lema 1.3 establece condiciones necesarias para que un espacio topologíco arbitrario sea conectificable.

Los resultados a considerar son aquellos que conservan los axiomas de separación al conectificar un espacio. Por ello se inicia con las siguientes definiciones.

\section{Definiciones:}

(a) Un espacio topológico $(X, \tau)$ es $T_{0}$ si para todo $x, y \in X, x \neq y$ existe un $V \in \tau$ tal que $|V \cap\{x, y\}|=1$. Si ocurre que existen $U, V \in \tau$ tales que $x \in V$ y y $\notin V$ y $y \in U$ y $x \notin U$, entonces se dice que $(X, \tau)$ es $T_{1}$; si además $U \cap V=\emptyset$ entonces, se dice que $X$ es un espacio $T_{2}$ o de Hausdorff.

(b) $\{U, V\}$ es una separación de $X$ si $U, V$ son abiertos ajenos cuya unión es $X .\{U, V\}$ es una separación trivial si $\{U, V\}$ es una separación tal que $U=\emptyset \circ V=\emptyset$.

(c) Se dice que $(X, \tau)$ es conexo si toda separación es trivial.

Se observa que un espacio $X$ es $T_{1}$ si y sólo si $\{x\}$ es cerrado para todo $x \in X$ y $X$ es de Hausdorff si y sólo si para todos $x, y \in X$ con $x \neq y$ existe un abierto $U \subset X$ tal que $x \in U$ y $y \notin c l_{X}(U)$. 
Definición: Un espacio topológico $(X, \tau)$ es conectificable si $X$ se puede sumergir densamente en un espacio $\left(Y, \tau^{*}\right)$ conexo. En este caso se dice que $\left(Y, \tau^{*}\right)$ es una conectificación de $(X, \tau)$.

Lema 1.1. Si $(X, \tau)$ es un espacio $T_{0}$, entonces $X$ tiene una conectificación $T_{0}$.

Demostración: Sea $(X, \tau)$ un espacio $T_{0}$ y $p \notin X$. Definimos $Y=X \cup\{p\}$ y $\tau^{*}=\tau \cup\{Y\}$; entonces, es fácil verificar que $\tau^{*}$ es una topología en $Y$ y que $X$ es denso en $Y$.

Para demostrar que $\left(Y, \tau^{*}\right)$ es conexo, tomemos $U, V \subseteq Y$ abiertos ajenos tales que $U \cup V=Y$. Si $p \in U$ entonces $U=Y$ y $V=\emptyset$. Si $p \in V$, entonces $V=Y$ así que $U=\emptyset$. Por lo tanto el espacio $\left(Y, \tau^{*}\right)$ es conexo.

Por último, se nota que $X$ es $T_{0}$ y abierto en $Y$ y por lo tanto dados $p, x \in Y,|X \cap\{p, x\}|=1$; así se tiene que $\left(Y, \tau^{*}\right)$ es $T_{0}$.

El Lema 1.1 establece condiciones necesarias y suficientes para que un espacio $T_{0}$ sea conectificable. Para obtener condiciones similares para espacios $T_{1}$, se requieren los siguientes dos lemas.

Lema 1.2. Si $(X, \tau)$ es un espacio $T_{1}$ sin puntos aislados, entonces cualquier abierto no vacio en $X$ es infinito.

Demostración: Para demostrar este hecho, asumimos que existe un abierto no vacío $U$ en $X$ el cual es finito; si $x \in U$ entonces $\{x\}$ es cerrado en $U$ puesto que $X$ es $T_{1}$, por lo tanto cualquier subconjunto de $U$ es cerrado en $U$, por ser $U$ finito; así que $U$ es un subespacio discreto de $X$. Ahora $U$ es abierto en $X$ y $\{x\}$ es abierto en $U$ por lo tanto $\{x\}$ es abierto en $X$ lo cual es imposible, pues $X$ no tiene puntos aislados. Así que, $U$ debe ser infinito.

Lema 1.3. Sea $X$ denso en $Y$ y $Y$ conexo. Si $C$ es abierto-cerrado en $X$, el cual es no vacío, entonces $C$ no es cerrado en $Y$.

Demostración: Suponiendo que $C$ es cerrado en $Y$ y tomando en cuenta que $X$ es denso en $Y$ tenemos:

$$
Y=c l_{Y}(X)=c l_{Y}(C \cup(X \backslash C))=c l_{Y}(C) \cup c l_{Y}(X \backslash C)=C \cup c l_{Y}(X \backslash C) .
$$


Pero $C \cap c l_{Y}(X \backslash C)=\emptyset$, pues en caso contrario existe $x \in C \cap c l_{Y}(X \backslash C)$, de donde se sigue que para todo abierto $U$ en $Y$ que contiene a $x$ se tiene $U \cap(X \backslash C) \neq \emptyset$ por lo tanto $(U \cap X) \cap(X \backslash C) \neq \emptyset$, es decir $x \in c_{X}(X \backslash C)=$ $X \backslash C$; entonces $x \notin C$, lo cual es una contradicción.

Así, se tiene que $C$ y $c l_{Y}(X \backslash C)$ forman una separación no trivial para el espacio $Y$, pero esto es imposible pues $Y$ es conexo. Así que, $C$ no es cerrado en $Y$.

Del lema anterior, se obtiene que si un espacio $X$ es conectificable, entonces no puede tener subconjuntos abierto-cerrados no vacíos que sean cerrados en la conectificación.

Corolario 1.4. Sea $Y$ un espacio conexo $T_{1}$. Si $X$ es denso en $Y$ entonces $X$ no tiene puntos aislados.

El siguiente resultado caracteriza completamente la conectificación de espacios $T_{1}$.

Lema 1.5. Si $(X, \tau)$ es un espacio $T_{1}$, entonces $(X, \tau)$ tiene una conectifcación $T_{1}$ si y sólo si $X$ no tiene puntos aislados.

Demostración: Si el espacio $X$ tiene una conectificación $T_{1}$, entonces por el Corolario $1.4, X$ no tiene puntos aislados.

$\Lambda$ hora si el cspacio no tiene puntos aislados, entonces $X$ es infinito. Construiremos una conectificación como sigue:

Sea $Y=X \cup\{p\}, p \notin X$. Diremos que $U \subset Y$ pertenece a $\tau^{*}$ si $U \in \tau$ ó $|(Y \backslash U)|<\omega$. Es fácil verificar que $\tau^{*}$ es una topología en $Y$.

Se afirma que $\left(Y, \tau^{*}\right)$ es conexo. Para demostrar esta afirmación, supongamos que $\{U, V\}$ es una separación de $Y$; entonces uno de los conjuntos $U, V$ contiene al punto $p$; supongamos que $p \in U$. Como $V=Y \backslash U$ tenemos que $|V|=|Y \backslash U|<\omega$, o sea que $V$ es finito. Además $p \notin V \in \tau^{*}$ y por lo tanto $V$ es abierto no vacío en $X$ así que por el Lema 1.2 el conjunto $V$ es infinito, lo cual es una contradicción. De modo que $Y$ es conexo. 
Por otra parte $Y$ es $T_{1}$ pues $X$ es $T_{1}$ y $\{p\}$ es cerrado en $Y$.

Finalmente, $X$ es denso en $Y$, pues $X$ es infinito y por lo tanto cada vecindad de $p$ tiene intersección no vacía con $X$.

De todo lo anterior se sigue que $\left(Y, \tau^{*}\right)$ es una conectificación $T_{1}$ del espacio $X$.

\section{Sección 2. Conectificación de espacios de Hausdorff y de Tychonoff numerables.}

Ya que hemos establecido condiciones necesarias y suficientes para que espacios $T_{0}$ y $T_{1}$ tengan conectificaciones $T_{0}$ y $T_{1}$ respectivamente, pasamos al caso de espacios de Hausdorff. Para el caso de espacios numerables de Hausdorff, una caracterización está dada en [19], pues ahí se demuestra que un espacio de Hausdorff numerable tiene una conectificación de Hausdorff si y solamente si no tiene puntos aislados. Condiciones suficientes para espacios numerables de Hausdorff también aparecen en [19], en particular se establecen condiciones suficientes para que un espacio de Hausdorff numerable tenga una conectificación de Hausdorff numerable y primero numerable. Una condición necesaria pero no suficiente está dada para espacios de Hausdorff en general.

En [1] se definió el concepto de $\omega$-conectificabilidad y se dieron condiciones suficientes para que espacios de Tychonoff numerables y espacios regulares numerables sean $\omega$-conectificables; en particular se demostró que todo espacio de Hausdorff numerable y denso en sí mismo con $\pi$-peso menor que el continuo es 'i-conectificable.

En la demostración del Teorema 1.11 se usará el hecho de que el espacio no tiene subconjuntos abiertos propios que sean débilmente compactos. Por tal motivo se necesitaran algunas caracterizaciones y propiedades de los espacios débilmente compactos.

Definiciones: Sea $X$ un espacio topológico.

(1) Dada una familia $\mathcal{F} \subseteq \mathcal{P}(X)$ tal que $\cup \mathcal{F}$ es denso en $X$, llamaremos a $\mathcal{F}$ sistema denso. Si $\mathcal{G} \subseteq \mathcal{F} y \cup \mathcal{G}$ es denso en $X$, entonces $\mathcal{G}$ se llama subsistema denso de $\mathcal{F}$. 
(2) Se dice que $(X, \tau)$ es débilmente compacto si y sólo si toda cubierta abierta y numerable de $X$ tiene un subsistema denso finito.

(3) Diremos que $(X, \tau)$ es un espacio $H$-cerrado si y sólo si toda cubierta abierta de $X$ tiene un subsistema denso finito.

(4) Se dice que una famila $\left\{U_{\alpha}: \alpha<\lambda\right\}$ de subconjuntos de $X$ es anidada si para cada $\alpha, \beta<\lambda U_{\alpha} \subseteq U_{\beta}$ si $\beta<\alpha$.

Teorema 1.6. Si $(X, \tau)$ es un espacio topológico, entonces $X$ es débilmente compacto si y sólo si toda familia numerable y anidada de abiertos no vacíos $\left\{U_{n}: n \in \omega\right\}$ tiene la propiedad $\cap\left\{\operatorname{cl}\left(U_{n}\right): n \in \omega\right\} \neq \emptyset$.

Demostración: Sea $(X, \tau)$ un espacio topológico débilmente compacto; supóngase que existe una familia anidada de abiertos no vacios $\left\{U_{n}: n \in \omega\right\}$ tal que $\cap\left\{c l\left(U_{n}\right): n \in \omega\right\}=\emptyset$. Entonces $\left\{X \backslash c l\left(U_{n}\right): n \in \omega\right\}$ es una cubierta abierta de $X$ y para cada $m \in \omega$ se tiene $\cup\left\{X \backslash c l\left(U_{n}\right): 1 \leq n \leq m\right\} \subseteq$ $X \backslash c l\left(U_{m+1}\right)$ el cual no es denso en $X$. Se sigue que $X$ no es débilmente compacto contradiciendo nuestra hipótesis.

Inversamente, supóngase que $X$ no es débilmente compacto. Entonces existc una cubierta abierta numerable de $X$, digamos $\mathcal{F}=\left\{V_{n}: n \in \omega\right\}$, que no posee ningún subsistema denso finito.

Ilagamos

$$
U_{1}=X \backslash c l\left(V_{1}\right), \ldots, U_{n}=X \backslash \operatorname{cl}\left(\cup\left\{V_{m}: 1 \leq m \leq n\right\}\right), \ldots
$$

Obsérvese que:

(1) $U_{n}$ es abierto para todo $n$;

(2) $U_{n+1}^{r} \subseteq U_{n}$

(3) $U_{n} \neq \emptyset$, ya que $U_{n}=\emptyset$ si y sólo si $c l\left(\cup\left\{V_{m}: 1 \leq m \leq n\right\}\right)=X$;

(4) $\operatorname{cl}\left(U_{n}\right) \subseteq X \backslash \cup\left\{V_{m}: 1 \leq m \leq n\right\}$;

(5) $\cap\left\{c l\left(U_{n}\right): n \in \omega\right\}=\emptyset$. 
De todo lo anterior vemos que existe una familia numerable y anidada $\left\{U_{n}: n \in \omega\right\}$ de abiertos no vacíos tales que $\cap\left\{c l\left(U_{n}\right): n \in \omega\right\}=\emptyset$ y la demostración está terminada.

Teorema 1.7. Un espacio $X$ es débilmente compacto si y sólo si toda familia localmente finita de abiertos mutuamente ajenos es finita.

Demostración: Sea $(X, \tau)$ débilmente compacto. Supongamos que existe $\mathcal{F}=\left\{U_{n}: n \in \omega\right\}$, la cual es una familia localmente finita de abiertos ajenos dos a dos tal que $\omega=|\mathcal{F}|$. Sean

$$
V_{0}=\cup\left\{U_{n}: n \in \omega\right\}, \ldots, V_{m}=\cup\left\{U_{n}: n \geq m\right\}, \ldots
$$

Entonces, $\left\{V_{n}: n \in \omega\right\}$ es una familia numerable y anidada de abiertos no vacíos tales que $\cap\left\{c l V_{n}: n \in \omega\right\}=\emptyset$ pues si no fuera así, existiría $x \in \cap\left\{c l V_{n}: n \in \omega\right\}$ o sea $x \in c l\left(U_{n}\right)$ para todo $n \in \omega$, lo cual es una contradicción pues $\mathcal{F}$ es una familia localmente finita. Así que $\mathcal{F}$ debe ser finita.

Inversamente, supóngase que $X$ no es débilmente compacto, entonces existe una familia numerable y anidada de abiertos no vacíos, digamos $\mathcal{F}=$ $\left\{U_{n}: n \in \omega\right\}$ tales que $\cap\left\{c l\left(U_{n}\right): n \in \omega\right\}=\emptyset$.

Definimos la familia $\left\{V_{n}: n \in \omega\right\}$ como sigue:

$$
V_{1}=X \backslash c l\left(U_{1}\right), \quad V_{2}=U_{1} \backslash c l\left(U_{2}\right), \ldots, V_{n}=U_{n-1} \backslash c l\left(U_{n}\right), \ldots
$$

Obsérvese que:

(1) Hay un numero infinito de $V_{n}$ no vacíos.

(2) Cada $V_{n}$ es abierto.

(3) La familia $\left\{V_{n}: n \in \omega\right\}$ es localmente finita, pues de otro modo existe $x \in X$ tal que para todo abierto $U$ que contiene a $x$, se tiene $U \cap V_{n} \neq \emptyset$ para un número infinito de $n \in \omega$, o sea $x \in \operatorname{cl}\left(V_{n}\right)$ para un número infinito de $n \in \omega$. Por lo tanto $x \in \cap\left\{c l U_{n}: n \in \omega\right\}=\emptyset$.

Así terminamos la demostración. 
Definiciones: Sea $(X, \tau)$ un espacio topológico.

(1) $(X, \tau)$ es un espacio Hausdorff minimal, si $\tau$ es una topología minima tal que $(X, \tau)$ es un espacio de Hausdorff, o sea si $\sigma \subset \tau$ y $\sigma \neq \tau$ entonces $(X, \sigma)$ no es de Hausdorff.

(2) Dado un $U \in \tau$, se dice que $U$ es un abierto regular si

$$
U=\operatorname{int}_{X}\left(c l_{X}(U)\right) .
$$

(3) La semirregularización de $(X, \tau)$ es el espacio $\left(X, \tau_{s}\right)$ donde $\tau_{s}$ es la topología generada por los abiertos regulares de $(X, \tau)$.

(4) Un espacio $(X, \tau)$ es de Katetov si existe $\tau^{*} \subset \tau$ tal que $\left(X, \tau^{*}\right)$ es Hausdorff minimal.

(5) Un espacio $(X, \tau)$ es disperso si cada subespacio de $(X, \tau)$ tiene un punto aislado.

Se sabe que un espacio compacto de Hausdorff es Hausdorff minimal y un espacio Hausdorff minimal es $H$-cerrado [7, Problema 3.12.5(e)] y además un espacio regular y $H$-cerrado es compacto. También se tiene que un espacio de Hausdorff es $H$-cerrado si y sólo si es cerrado como subespacio de cualquier espacio de Hausdorff [7, Problema 3.12.5(a)]. De este hecho y del Lema 1.3 se tiene que un espacio de Hausdorff conectificable no contiene subconjuntos abierto-cerrados no vacíos que sean $H$-cerrados, esta condición no es suficiente como se demuestra en la Sección 4 de [19].

Corolario 1.8. Sea $(X, \tau)$ un espacio de Hausdorff y numerable; $X$ es $H$ cerrado si y sólo si toda familia ajena dos a dos de abiertos localmente finita en $X$ es finita.

Demostración: Puesto que un espacio numerable es $H$-cerrado si y sólo si es débilmente compacto y esto sucede si y sólo si toda familia localmente finita de abiertos mutuamente ajenos es finita.

Lema 1.9. Todo espacio topológico $H$-cerrado y numerable es disperso. 
Demostración: Sea $(X, \tau)$ un espacio $H$-cerrado y numerable. La semirregularización de un espacio $H$-cerrado es minimal Hausdorff [7, Problema 3.12.5(e)]. Por lo tanto $X$ es de Katetov. Pero todo espacio de Katetov y numerable es disperso [14, Teorema 5.2]. Por lo tanto $X$ es disperso.

Definiciones: Sea $\mathcal{F} \subseteq \mathcal{P}(X)$. Se dice que $\mathcal{F}$ es un filtro abierto si $\mathcal{F}$ es un filtro con una base de abiertos. $\mathcal{F}$ es un filtro abierto libre $\operatorname{si} \cap\{c l(F): F \in$ $\mathcal{F}\}=\emptyset$. Además $\mathcal{F}$ es un ultrafiltro abierto si es un filtro abierto el cual es maximal. Por otro lado, $\mathcal{F}$ es un ultrafiltro abierto libre si es un ultrafiltro abierto tal que $\cap\{c l(F): F \in \mathcal{F}\}=\emptyset$. Un punto $x \in X$ es de acumulación para el filtro $\mathcal{F}$, si toda vecindad de $x$ intersecta a todos los elementos de $\mathcal{F}$; si toda vecindad de $x$ pertenece a $\mathcal{F}$, entonces se dice que $\mathcal{F}$ converge a $x$.

Lema 1.10. Si $U$ es un conjunto abierto no vacío de $X$, cuya cerradura no es débilmente compacta, entonces $U$ es un elemento de por lo menos $2^{\mathfrak{C}}$ ultrafiltros abiertos libres distintos en $X$.

Demostración: Sea $(X, \tau)$ un espacio topológico y $U$ un abierto no vacío tal que $c l(U)$ no es débilmente compacto. Dado que $c l(U)$ no es débilmente compacto, por el Teorema 1.7 existe una familia infinita y localmente finita de abiertos mutuamente ajenos digamos $\left\{U_{n}: n \in \omega\right\}$, donde $U_{n} \subseteq U$ para cada $n \in \omega$.

Ahora, dado un ultrafiltro libre $\mathcal{F}$ en $\omega$, se induce un filtro abierto libre en $X$, denotado por $\mathcal{F}^{*}$ y definido como sigue: $G \in \mathcal{F}^{*}$ si y sólo si se cumplen las siguientes condiciones:

(i) $G$ es abierto en $X$ y

(ii) existe $F \in \mathcal{F}$ tal que $\cup\left\{U_{n}: n \in F\right\} \subseteq G$.

Veamos que $\mathcal{F}^{*}$ es un filtro abierto sobre $X$.

(i) Como $\emptyset \neq U_{n}$ para todo $n \in \omega$, entonces $\emptyset \notin \mathcal{F}^{*}$.

(ii) Sean $H$ y $G$ elementos de $\mathcal{F}^{*}$, entonces $H$ y $G$ son abiertos en $X$ y existen $F_{1}$ y $F_{2}$ en $\mathcal{F}$ tales que

$$
\cup\left\{U_{n}: n \in F_{1}\right\} \subseteq H \quad \text { y } \quad \cup\left\{U_{n}: n \in F_{2}\right\} \subseteq G .
$$


Por lo tanto $H \cap G$ es abierto en $X$ y $\cup\left\{U_{n}: n \in F_{1} \cap F_{2}\right\} \subseteq H \cap G$. Así que, $H \cap G \in \mathcal{F}^{*}$.

(iii) Sean $H \in \mathcal{F}^{*}$ y $G \subseteq U$ tales que $H \subseteq G$ y $G$ abierto en $X$, entonces $H$ cs abierto en $X$ y existe $F \in \mathcal{F}$ tal que $\cup\left\{U_{n}: n \in F\right\} \subseteq H \subseteq G$. Por lo tanto $G \in \mathcal{F}^{*}$. Así que, $\mathcal{F}^{*}$ es un filtro abierto en $X$.

Afirmamos que $\mathcal{F}^{*}$ es libre; si no, entonces $\cap\left\{\operatorname{cl}(G): G \in \mathcal{F}^{*}\right\} \neq \emptyset$ y por lo tanto existe $x \in c l(G)$ para todo $G \in \mathcal{F}^{*}$. Así que para todo $V$ abierto en $X$ que contiene a $x$ se tiene $V \cap G \neq \emptyset$ para toda $G \in \mathcal{F}^{*}$. En particular $V \cap\left(\cup\left\{U_{n}\right): n \in \omega\right\} \neq \emptyset$ lo cual implica que $V \cap U_{n} \neq \emptyset$ para algún $n \in \omega$, pero esto es contradictorio ya que $\left\{U_{n}: n \in \omega\right\}$ es una familia localmente finita.

Por otro lado, la correspondencia $\mathcal{F} \rightarrow \mathcal{F}^{*}$ es inyectiva; pues si tenemos $\mathcal{F}_{1}$ y $\mathcal{F}_{2}$ ultrafiltros libres distintos sobre $\omega$, entonces existen $F_{1} \in \mathcal{F}_{1}$ y $F_{2} \in \mathcal{F}_{2}$ ajenos y por lo tanto

$$
G-\cup\left\{U_{n}: n \in F_{1}\right\} \in \mathcal{F}_{1}^{*} \quad \text { y } \quad H=\left\{U_{n}: n \in F_{2}\right\} \in \mathcal{F}_{2}^{*}
$$

son elementos ajenos de $\mathcal{F}_{1}^{*}$ y $\mathcal{F}_{2}^{*}$ respectivamente; así que $\mathcal{F}_{1}^{*} \neq \mathcal{F}_{2}^{*}$. Ahora, sca $\mathcal{U}_{\mathcal{F}}$ un ultrafiltro abierto que conticne a $\mathcal{F}^{*}$ entonces, la correspondencia $\mathcal{F} \rightarrow \mathcal{U}_{\mathcal{F}}$ es inyectiva y puesto que hay $2^{\mathfrak{C}}$ ultrafiltros en $\omega$, el resultado queda demostrado.

El siguiente resultado, demostrado en [19], da una caracterización para que los espacios de Hausdorff numerables tengan una conectificación de Hausdorff. La conectificación del espacio $X$ se obtiene agregando puntos que no pertenecen a $X$ y definiendo las vecindades de estos puntos en términos de ultrafiltros en $X$.

En lo que sigue necesitaremos las siguientes definiciones.

Definiciones: Sea $(X, \tau)$ un espacio topológico.

(1) Se dice que $\mathcal{B} \subset \tau \backslash\{\emptyset\}$ es una $\pi$-base de $X$ si para todo $U \in \tau \backslash\{\emptyset\}$ existe $B \in \mathcal{B}$ tal que $B \subset U$. 
(2) Se define el peso del espacio $X$ como $w(X)=\min \{|\mathcal{B}|: \mathcal{B}$ es una base de $X\}+\omega$.

(3) Se define el $\pi$-peso del espacio $X$ como $\pi w(X)=\min \{|\mathcal{B}|: \mathcal{B}$ es una $\pi$-base de $X\}+\omega$.

(4) Sea $x \in X$; se define el carácter de $x$ como $\chi(x, X)=\min \{|\mathcal{B}|: \mathcal{B}$ es una base local para $x\}+\omega$.

(5) Se define la densidad del espacio $X$ comod $(X)=\min \{|D|: D$ es denso en $X\}+\omega$.

Teorema 1.11. Sea $(X, \tau)$ un espacio de Hausdorff y numerable. $X$ tiene una conectificación de Hausdorff si y sólo si no tiene puntos aislados.

Demostración: Si $X$ tiene una conectificación de Hausdorff, entonces por el Lema 1.5 no tiene puntos aislados.

Inversamente, supóngase que $X$ no tiene puntos aislados. Se afirma que ningún subespacio propio y abierto de $X$ es débilmente compacto. Pues si existe un abierto no vacío $U \subset X$ tal que $U$ es débilmente compacto, entonces $U$ es numerable y de Hausdorff. Así que por el Corolario 1.8, $U$ es $H$-cerrado y por el Lema 1.9, $U$ es disperso; o sea $U$ tiene puntos aislados, los cuales son aislados en $X$ pues $U$ es abierto, pero esto es una contradicción.

Sea $\mathcal{B}$ una $\pi$-base para $X$; obviamente $|\mathcal{B}| \leq \mathfrak{c}$. Por el argumento del párrafo anterior $c l(B)$ no es débilmente compacto para todo $B \in \mathcal{B}$. Así que por el Lema $1.10, B$ es un elemento de al menos $2^{\mathfrak{C}}$ ultrafiltros abiertos libres distintos en $X$.

Sea $\mathcal{P}=\{\{B, C\}: B, C \in \mathcal{B}$ y $B \cap C=\emptyset\}$ y bien ordenamos $\mathcal{P}$ como $\left\{P_{\alpha}: \alpha<\lambda\right\}$ donde $P_{\alpha}=\left\{B_{\alpha}, C_{\alpha}\right\}$; obviamente $\lambda \leq \mathfrak{c}$.

Para cada $\alpha<\lambda$ sea $p_{\alpha} \notin X$. Además, suponemos que $p_{\alpha} \neq p_{\beta}$ si $\alpha \neq \beta$, $\alpha, \beta<\lambda$. Escogemos ultrafiltros abiertos libres $\mathcal{U}_{0}$ y $\mathcal{V}_{0}$ tales que $B_{0} \in \mathcal{U}_{0}$ y $C_{0} \in \mathcal{V}_{0}$. Sea $\beta<\lambda$, al haber escogido ultrafiltros abiertos libres $\mathcal{U}_{\alpha}$ y $\mathcal{V}_{\alpha}$ para cada $\alpha<\beta$ escogemos ultrafiltros $\mathcal{U}_{3}$ y $\mathcal{V}_{3}$ como sigue: puesto que $B_{\beta}$ y $C_{3}$ no son débilmente compactos, pertenecen cada uno a al menos $2^{\mathfrak{c}}$ 
ultrafiltros abiertos libres distintos. Sean $\mathcal{U}_{\beta}$ y $\mathcal{V}_{\beta}$ ultrafiltros abiertos libres tales que $B_{\beta} \in \mathcal{U}_{\beta}, C_{\beta} \in \mathcal{V}_{\beta}$ y $\mathcal{U}_{\beta} \neq \mathcal{U}_{\alpha}, \mathcal{V}_{\beta} \neq \mathcal{V}_{\alpha}$ para cada $\alpha<\beta$.

Sea $Y=X \cup\left\{p_{\alpha}: \alpha<\lambda\right\}$. Definimos una base $\mathcal{D}$ de una topología $\tau^{*}$ en $Y$ como sigue:

(1) Un conjunto $U \subseteq X$ es abierto en $Y$ si y sólo si es abierto en $X$; en este caso $U \in \mathcal{D}$;

(2) Las vecindades abiertas básicas de $p_{\alpha}$ (pertenecientes a $\mathcal{D}$ ) son todos los conjuntos de la forma $\left\{p_{\alpha}\right\} \cup U \cup V$ don'́e $U \in \mathcal{U}_{\alpha}$ y $V \in \mathcal{V}_{\alpha}$.

Fl espacio $X$ es denso en $Y$. En efecto, dado $\emptyset \neq W \in \mathcal{D}$ hay dos casos:

(a) $W \in \tau$. Entonces $W \cap X \neq \emptyset$.

(b) $W=\left\{p_{\alpha}\right\} \cup U \cup V$ donde $U \in \mathcal{U}_{\alpha}$ y $V \in \mathcal{V}_{\alpha}$. Entonces $W \cap X=$ $U \cup V \neq \emptyset$ pues $U$ y $V$ son subconjuntos no vacios de $X$ y $p_{\alpha} \notin X$. Por lo tanto $W \cap X \neq \emptyset$.

Lo anterior implica que $X$ es denso en $Y$.

Se afirma que $Y$ es de Hausdorff. Para ver esto tomemos dos puntos distintos de $Y$. Hay tres casos:

a) Los dos puntos están en $X$. Entonces existen dos abiertos en $X$ y por lo tanto abiertos en $Y$, los cuales son ajenos y que separan los dos puntos.

b) Los puntos son $x \in X$ y $p_{\alpha} \in Y \backslash X$. Como $x \in X$ entonces $x$ no es punto de acumulación de $\mathcal{U}_{\alpha}$ ni de $\mathcal{V}_{\alpha}$; así que existe una vecindad $W$ de $x$ la cual es ajena a algún elemento de $\mathcal{U}_{\alpha}$ digamos $U_{\alpha}$ y ajena a $V_{\alpha} \in \mathcal{V}_{\alpha}$. Por lo tanto $\left[\left\{p_{\alpha}\right\} \cup U_{\alpha} \cup V_{\alpha}\right] \cap W \neq \emptyset$. Así que $x$ y $p_{\alpha}$ pertenecen a conjuntos abiertos de $Y$ que son mutuamente ajenos.

c) Los puntos son $p_{\alpha}$ y $p_{\beta}$ con $\alpha \neq \beta$. Entonces existen elementos ajenos digamos $U, V, W, Z$ de $\mathcal{U}_{\alpha}, \mathcal{V}_{\alpha}, \mathcal{U}_{\beta}$ y $\mathcal{V}_{\beta}$ respectivamente, tales que $\left[\left\{p_{\alpha}\right\} \cup\right.$ $U \cup V] \cap\left[\left\{p_{\beta}\right\} \cup W \cup Z\right]=\emptyset$. Así que $p_{\alpha}$ y $p_{\beta}$ se pueden separar por abiertos ajenos de $Y$. 
De los incisos anteriores resulta que $Y$ es de Hausdorff.

Finalmente veamos que $Y$ es conexo. Supóngase que no. Entonces existe una separación $\{S, T\}$ de $Y$. Se sigue que $S \cap X$ y $T \cap X$ son abierto-cerrados ajenos en $X$, y por lo tanto existe $\alpha<\lambda$ tal que $B_{\alpha} \subseteq S \cap X$ y $C_{\alpha} \subseteq T \cap X$, pues $\mathcal{B}$ es una $\pi$-base de $X$. Por lo tanto, $p_{\alpha} \in \operatorname{cl}\left(B_{\alpha}\right) \cap \operatorname{cl}\left(C_{\alpha}\right) \subseteq S \cap T$. Se sigue que $S \cap T \neq \emptyset$ lo cual es una contradicción.

De todo lo anterior tenemos que $Y$ es una conectificación de Hausdorff para $X$.

Se observa, que en la demostración anterior si $X$ tiene una $\pi$-base numerable, entonces $|Y \backslash X|=\omega$ y por lo tanto la conectificación $Y$ es numerable con $\pi$-peso numerable, tal como se muestra en el siguiente corolario.

Corolario 1.12. Todo espacio de Hausdorff numerable con un $\pi$-peso numerable y sin puntos aislados, tiene una conectificación de Hausdorff con $\pi$-peso numerable.

Demostración: Sea $(X, \tau)$ un espacio de Hausdorff numerable y sin puntos aislados. Por cl Tcorema $1.11 X$ tiene una conectificación $Y$ la cual es de Hausdorff. Observemos que toda $\pi$-base de $X$ induce una $\pi$-base de $Y$, pues $X$ es abierto $y$ denso en $Y$. Por lo tanto, si $X$ tiene $\pi$-peso numerable, entonces $Y$ tiene $\pi$-peso numerable.

Lema 1.13. Si $U$ es un subconjunto abierto no vacío de $X$, cuya cerradura no es débilmente compacta, entonces $U$ es un elemento de un filtro abierto iibre en $X$, con base numerable.

Demostración: Dado que $c l(U)$ no es débilmente compacto, por el Teorema 1.7 existe una familia localmente finita de subconjuntos abiertos no vacíos y mutuamente ajenos de $U$, digamos $\left\{U_{n}: n \in \omega\right\}$. Sea $B_{n}=\cup\left\{U_{\kappa}: n \leq \kappa\right\}$, tomemos $\mathcal{B}=\left\{B_{n}: n \in \omega\right\}$ la cual es una base numerable para un filtro abierto libre sobre $X$, que contiene a $U$. 
En el siguiente teorema, que aparece en [19], se construye una conectificación de $X$ agregando puntos que no pertenecen a $X$ y definiendo las vecindades de estos puntos, en términos de elementos de ciertos filtros abiertos libres en $X$.

Teorema 1.14. Sea $(X, \tau)$ un espacio de Hausdorff, numerable, primero numerable y sin puntos aislados, entonces $X$ tiene una conectificación de Hausdorff, numerable y primero numerable.

Demostración: Como en el Teorema 1.11, se puede demostrar que $X$ no tiene ningún subespacio abierto, cuya cerradura sea débilmente compacta.

Sea $\mathcal{B}$ una base numerable para $X$; dicha base existe pues $X$ es numerable y primero numerable. Sea $\mathcal{P}=\left\{P_{n}: n \in \omega\right\}$, un buen ordenamiento del conjunto de todos los pares $P_{n}=\left\{B_{n}, C_{n}\right\}$ de elementos no vacíos ajenos de $\mathcal{B} \mathrm{y}\left\{p_{n}: n \in \omega\right\}$ un conjunto de puntos distintos tales que $p_{n} \notin X$ para todo $n \in \omega$. Definimos una topología en el espacio $Y=X \cup\left\{p_{n}: n \in \omega\right\}$ como sigue:

(i) $U \subseteq X$, es abierto en $Y$ si y sólo si $U$ es abierto en $X$;

(ii) Las vecindades de $p_{n}$ se definen como sigue por inducción: Consideremos $P_{1}=\left\{B_{1}, C_{1}\right\}$; puesto que $B_{1}$ y $C_{1}$ son abiertos no vacíos, por la observación dada al principio, las cerraduras de estos conjuntos no son débilmente compactos, así que por el Lema 1.13 existen filtros abiertos libres $\mathcal{U}_{1}$ y $\mathcal{V}_{1}$ en $X$ con bases numerable tales que $B_{1} \in \mathcal{U}_{1}$ y $C_{1} \in \mathcal{V}_{1}$. Una vecindad de $p_{1}$ es un conjunto de la forma $\left\{p_{1}\right\} \cup U \cup V$ con $U \in \mathcal{U}_{1}$ y $V \in \mathcal{V}_{1}$.

Al haber definido las vecindades de $p_{1}, p_{2}, \ldots, p_{n-1}$ en $Y$, consideremos $P_{n}=\left\{B_{n}, C_{n}\right\}$. Tomemos $x \in B_{n}$; dado que $x$ no es punto de acumulación de $\mathcal{U}_{1}, \mathcal{U}_{2}, \ldots, \mathcal{U}_{n-1}, \mathcal{V}_{1}, \mathcal{V}_{2}, \ldots, \mathcal{V}_{n-1}$ existen vecindades abiertas $S_{k}$ y $T_{k}$ de $x$ con $1 \leq k \leq n-1$ tales que $S_{k}$ no intersecta a algún elemento de $\mathcal{U}_{k}$ y además $T_{k}$ no intersecta a algún elemento de $\mathcal{V}_{k}$. Sea $B_{n}^{*}=\cap\left\{S_{k} \cap T_{k}: 1 \leq k \leq\right.$ $n-1\} \subset B_{n}$. Entonces $B_{n}^{*}$ es un abierto no vacío en $X$ y $B_{n}^{*} \subseteq B_{n}$. Dado que $S_{k}$ y $T_{k}$ no intersectan a algún elemento de $\mathcal{U}_{k}$ y $\mathcal{V}_{k}$ respectivamente, se sigue que $B_{n}^{*}$ es ajeno a algún elemento de $\mathcal{U}_{k}$ y algún elemento de $\mathcal{V}_{k}$ respectivamente, para todo $1 \leq k \leq n-1$. Por ser $B_{n}^{*}$ abierto no vacío, no es 
débilmente compacto, así que por el Lema 1.13 existe un filtro abierto libre $\mathcal{U}_{n}$ sobre $X$, con base numerable, que contiene a $B_{n}^{*}$. Procediendo de la misma forma, obtenemos un filtro abierto libre $\mathcal{V}_{n}$ sobre $X$, con base numerable, que contiene a $C_{n}^{*}$. Tomemos las vecindades abiertas de $\left\{p_{n}\right\}$, como los conjuntos de forma $\left\{p_{n}\right\} \cup U \cup V$ donde $U \in \mathcal{U}_{n}$ y $V \in \mathcal{V}_{n}$.

La demostración de que $\left(Y, \tau^{*}\right)$ es un espacio topológico de Hausdorff y conexo tal que $X$ es denso en $Y$, es similar a la dada en el Teorema 1.11 y $Y$ es obviamente numerable y segundo numerable.

No se conocen condiciones necesarias y suficientes para caracterizar conectificaciones de espacios numerables de Tychonoff, sin embargo condiciones suficientes están dadas en [1].

Nuestro propósito en lo que sigue es extender el Corolario 1.12 a espacios numerables cuyo $\pi$-peso es menor que $\mathfrak{c}$. Para ello necesitaremos algunas definiciones $y$ resultados.

Definiciones: Sea $X$ un espacio topológico. Si $\left\{Y_{i}: i \in J\right\}$ y $\mathcal{F}=\left\{f_{i}: i \in\right.$ $J\}$ son una familia de espacios topológicos y una familia de funciones tales que $f_{i}: X \rightarrow Y_{i}$, entonces:

(a) $\mathcal{F}$ separa puntos de $X$, si para cada par de puntos distintos $x, y \in X$ existe una función $f_{i} \in \mathcal{F}$ tal que $f_{i}(x) \neq f_{i}(y)$.

(b) $\mathcal{F}$ separa puntos de cerrados, si para todo $x \in X$ y todo subconjunto cerrado $F \subset X$ tal que $x \notin F$, existe una función $f_{i} \in \mathcal{F}$ tal que $f_{i}(x) \notin c l_{X} f_{i}[F]$.

(c) Se define la función diagonal $f: X \rightarrow \prod_{i \in J} Y_{i}$ mediante la fórmula $f(x)=\left(f_{i}(x)\right)_{i \in J}$.

(d) Se dice que $X$ es perfectamente normal, si $X$ es normal y todo subconjunto cerrado de $X$ es un $G_{\delta}$.

Se observa que la función diagonal $f$ es continuo si cada $f_{i}$ es continuo y $f$ es inyectivo si $\mathcal{F}$ separa puntos. Además si $\mathcal{F}$ separa puntos de cerrados entonces $f$ es una inmersión en el producto $\prod_{i \in J} Y_{i}$. 
Proposición 1.15. Sea $(X, \tau)$ un espacio regular numerable con una $\pi$-base $\gamma$. Entonces existe una topología $\tau^{*}$ en $X$ tal que $\left(X, \tau^{*}\right)$ es un espacio $T_{3}$ con $\gamma \subseteq \tau^{*} \subseteq \tau$ y $w\left(X, \tau^{*}\right) \leq|\gamma| \cdot \omega$.

Demostración: Dado que $(X, \tau)$ es regular y numerable, el espacio $X$ es normal y todo subconjunto de $X$ es un $G_{\delta}$, por lo tanto $X$ es perfectamente normal. De aquí que para todo $V \in \tau$ existe una función continua $f_{V}: X \rightarrow$ $[0,1]$ tal que $X \backslash V=f_{V}^{-1}(0)$. Sea $f$ la función diagonal de las funciones $f_{V}$ con $V \in \delta=\gamma \cup \mu$, donde $\mu$ es una familia numerable de abiertos en $X$ que separa puntos de $X$. Es claro que $f$ es una función continua e inyectiva de $X^{-}$en $[0,1]^{\delta}$ y $|\delta| \leq|\gamma| \cdot \omega$. Tomemos una base $\mathcal{B}$ de $[0,1]^{\delta}$ tal que $|\mathcal{B}| \leq|\delta|$. Sea $\tau^{*}$ la topología en $X$ generada por $\left\{f^{-1}(U): U \in \mathcal{B}\right\}$. Esta topología cumple con las propiedades deseadas. En efecto, $\tau^{*}$ es la topología débil de $X$ con respecto a $f$ por lo tanto $\tau^{*} \subseteq \tau$ y además, $\gamma \subset \tau^{*}$ pues si $V \in \gamma$ cntonces existe una función continua $f_{V}: X \rightarrow[0,1]$ tal que $X \backslash V=f_{V}^{-1}(0)$. Sea $B=\Pi_{W^{\prime} \in \delta} J_{W^{\prime}}$ donde $J_{W}=(0,1]$ si $W=V$ y $J_{W}=[0,1]$ si $W \neq V$. Tenemos que $V=f_{V}^{-1}(B)$ y siendo que $B$ es un abierto en $[0,1]^{\delta}$ se sigue que $V \in \tau^{*}$. Además $w\left(X, \tau^{*}\right) \leq|\mathcal{B}| \leq|\delta| \leq|\gamma| \cdot \omega$.

Lema 1.16. Sea $X$ un espacio semirregular. Supóngase que $D$ es denso en $X, x \in D$ y $\lambda(x, D)=\lambda$. Entonces $\chi(x, X)=\lambda$.

Demostración: Sea $\left\{V_{\alpha}: \alpha<\lambda\right\}$, una base local de $x$ en $D$ y $U$ un abierto regular en $X$ tal que $x \in U$. Entonces $U \cap D$ es una vecindad de $x$ en $D$ y por lo tanto existe $\alpha \in \lambda$ tal que $x \in V_{\alpha} \subseteq U \cap D$, de donde se tiene que $c l_{X}\left(V_{\alpha}\right) \subseteq c l_{X}(U \cap D)=c l_{X}(U)$. Ahora $V_{\alpha}=U_{\alpha} \cap D$ para algún $U_{\alpha}$ abierto en $X$; entonces $c l_{X} V_{\alpha}=c l_{X}\left(U_{\alpha} \cap D\right)=c l_{X} U_{\alpha} \supset U_{\alpha}$ por lo tanto $x \in \operatorname{int}_{X}\left(c l_{X} V_{\alpha}\right)$ y se sigue que $x \in \operatorname{int}_{X}\left(c l_{X}\left(V_{\alpha}\right)\right) \subseteq \operatorname{int}_{X}\left(c l_{X}(U)\right)=U$, o sea $\left\{\operatorname{int}_{X}\left(c l_{X}\left(V_{\alpha}\right)\right): \alpha<\lambda\right\}$ es una base local de $x$ en $X$. Se concluye que $\chi(x, X)=\lambda$ si $\chi(x, D)=\lambda$.

\section{Definiciones:}

a) Una familia $\mathcal{A}$ de subconjuntos de $\omega$ tiene la propiedad de la intersección finita fuerte (piff), si para cualquier $\mathcal{B} \subset \mathcal{A}$ finito, se tiene que $\cap \mathcal{B}$ es infinito. 
b) Sea $\mathcal{A}$ una familia de subconjuntos de $\omega$ decimos que $B \subset \omega$ es una pseudointersección de $\mathcal{A}$, si $B \backslash A$ es finito para todo $A \in \mathcal{A}$.

c) El cardinal $\mathfrak{p}$ es la mínima potencia de una subfamilia de subconjuntos infinitos de $\omega$ con piff, la cual no tiene pseudointerseccion infinita.

El siguiente lema (Lema de Booth) será usado de forma frecuente en lo que sigue. Se sabe que este lema es independiente de los Axiomas de ZFC.

Lema 1.17. Lema de Booth (BL). $\mathfrak{p}=\mathfrak{c}$.

El lema anterior aparece en [16]. Para nuestro trabajo se requiere la siguiente equivalencia:

Sea $\mathcal{A}, \mathcal{B}$ familias de subconjuntos de $\omega$ tales que

1) $|\mathcal{A}|<\mathfrak{e},|\mathcal{B}|<\mathfrak{e}$

2) $|B \backslash \cup \mathcal{F}| \geq \omega$ para cada $B \in \mathcal{B}$ y cada subfamilia finita $\mathcal{F} \subset \mathcal{A}$.

Entonces existe $C \subset \omega$ tal que $C \cap B$ es infinita para cada $B \in \mathcal{B}$ y $C \cap A$ es finita para cada $A \in \mathcal{A}$.

Lema 1.18. (BL). Si $(X, r)$ es un espacio regular numerable sin puntos aislados y $w(X)<\mathfrak{c}$, entonces $\beta X \backslash X$ es separable y denso en $\beta X$.

Demostración: Por un teorema de Eda, Kamo y Nogura [5] y bajo el Lema de Booth, todo espacio regular numerable y no disperso con $w(X)<\mathfrak{c}$ contiene una copia topológica de los racionales.

Por el Lema de Zorn, la familia de todas las familias de copias mutuamente ajenas de los racionales en $X$ tiene un elemento máximo $\gamma$. Sea $Z=U \gamma$; si $U$ es abierto en $X$ entonces $U \cap Z \neq \emptyset$ pues si no fuera así, $U$ contendría una copia $Q$ de los racionales y $\gamma \cup\{Q\} \supset \gamma$ y $\gamma \cup\{Q\} \neq \gamma$ lo que contradice la maximalidad de $\gamma$. Por lo tanto $Z$ es denso en $X$. Claramente $|Z| \leq|X|=\omega$.

Sean $z \in Z$ y $C \in \gamma$ tales que $z \in C$ y $K=c_{\beta X}(C)$. Entonces, por el Lema $1.16 \mathrm{y}$ por ser $C$ primero numerable se tiene que $\chi(z, K)=\chi(z, C)=\omega$. Además, $K \backslash X$ es denso en $K$ pues $K$ es una compactificación de la copia $C$ de los racionales y el conjunto de los racionales no es localmente compacto en 
ningún punto. Se sigue que existe una sucesión $S_{z} \subseteq K \backslash X$ la cual converge a $z$. Sea $D=\cup\left\{S_{z}: z \in Z\right\}$; entonces $D$ es un subconjunto numerable de $\beta X \backslash X$ el cual es denso en $\beta X$. Por lo tanto $\beta X \backslash X$ es separable.

Definición: Si existe una conectificación $Y$ de $X$, tal que si $Y \backslash X$ es numerable, entonces se dice que $X$ es $\omega$-conectificable o numerablemente conectificable.

Se observa que el Corolario 1.12 y el Teorema 1.14 proporcionan ejemplos de espacios $\omega$-conectificables.

En lo que sigue $T(X)$ denotará la topología de $X, T(x, X)$ denotará los conjuntos abiertos en $X$ que contienen a $x$ y $T(F, X)$ denotará los abiertos en $X$ que contienen a $F \subseteq X$.

En la siguiente proposición, que aparece en [1], se construye una conectificación del espacio $X$ agregándole puntos que no pertenecen a $X$ y definiendo sus vccindades en términos de abiertos en una compactificación $c X$ de $X$.

Proposición 1.19. Sea $X$ un espacio de Tychonoff, numerable y denso en si. Supóngase que $X$ tiene una compactificación $c X$ para la cual existen conjuntos compactos $F_{n}, n \in \omega$, tales que:

(1) $F_{n} \cap F_{m}=\emptyset$ para $n \neq m$;

(2) $F_{n} \subseteq c X \backslash X$ para todo $n \in \omega$;

(3) Para todo par $(U, V)$ de subconjuntos abiertos no vacios y ajenos de $c X$, con $U \cup V$ denso en $c X$, existe $n \in \omega$ con $F_{n} \cap U \neq \emptyset \neq F_{n} \cap V$;

Entonces $X$ es $\omega$-conectificable.

Demostración: Sea $Y=X \cup\left\{y_{n}: n \in \omega\right\}$ donde ningún $y_{n}$ pertenece a $X$. La topología $\tau$ en $Y$ se define como sigue:

Todo abierto en $X$ es abierto en $Y$ y una base de vecindades abiertas de $y_{n}$ consiste de conjuntos $O_{n}(W)=\left\{y_{n}\right\} \cup\{W \cap X\}$, donde $W \in T\left(F_{n}, c X\right)$.

Para demostrar que el espacio $(Y, \tau)$ es de Hausdorff, consideramos tres casos: 
(1) Sean $x, y \in X$ con $x \neq y$. El espacio $X$ es de Tychonoff y por lo tanto de Hausdorff, es decir $x, y$ se pueden separar por abiertos ajenos en $X$, los cuales son abiertos en $Y$.

(2) Si $n \in \omega$ y $x \in X$, entonces dado que $c X$ es de Hausdorff y $F_{n}$ compacto, existen $W \in T\left(F_{n}, c X\right)$ y $U \in T(x, c X)$ tales que $W \cap U=\emptyset$. De aqui $O_{n}(W) \cap(U \cap X)=\emptyset$. Por lo tanto $y_{n}$ y $x$ se pueden separar por abiertos ajenos de $Y$.

(3) Sean $m, n \in \omega$ y $n \neq m$. Como $c X$ es de Hausdorff y $F_{n}$ y $F_{m}$ son compactos, existen $W_{1} \in T\left(F_{n}, c X\right)$ y $W_{2} \in T\left(F_{m}, c X\right)$ tales que $W_{1} \cap$ $W_{2}=\emptyset$. Entonces $O_{n}\left(W_{1}\right) \cap O_{m}\left(W_{2}\right)=\emptyset \mathrm{y}$ por lo tanto $y_{n} \mathrm{y} y_{m}$ se pueden separar por abiertos ajenos de $Y$.

Los incisos anteriores prueban que $Y$ es de Hausdorff.

Además $X$ es denso en $Y$ y $Y \backslash X$ es numerable por construcción.

Veamos que $Y$ es conexo. Supóngase que existe un abierto-cerrado $O$ en $Y$ tal que $O \neq \emptyset$ y $O \neq Y$. Los conjuntos $O_{1}=O \cap X$ y $X \backslash O_{1}$ son abiertocerrados en $X$, así que $c l_{c X}\left(O_{1}\right)=U$ y $c l_{c X}\left(X \backslash O_{1}\right)=V$ son abierto-cerrados ajenos en $c X$. Por el inciso (3) existe $n \in \omega$ tal que $F_{n} \cap U \neq \emptyset \neq F_{n} \cap V$ y en consecuencia

$$
F_{n} \cap c l_{c X}\left(O_{1}\right) \neq \emptyset \neq F_{n} \cap c l_{c X}\left(X \backslash O_{1}\right) .
$$

Por lo tanto, si $W \in T\left(F_{n}, c X\right)$ entonces $W \cap O_{1} \neq \emptyset \neq W \cap\left(X \backslash O_{1}\right)$. Se sigue que $O_{n}(W) \cap O_{1} \neq \emptyset$ y $O_{n}(W) \cap\left(X \backslash O_{1}\right) \neq \emptyset$ para todo $W \in T\left(F_{n}, c X\right)$, de donde $y_{n} \in \operatorname{cl}_{Y}\left(O_{1}\right) \cap c l_{Y}\left(X \backslash O_{1}\right)=\emptyset$ lo cual es una contradicción. Se sigue que $Y$ es conexo.

De todo lo anterior se tiene que $X$ es $\omega$-conectificable.

Definición: La familia de conjuntos compactos $\left\{F_{n}: n \in \omega\right\}$ con las propiedades enunciadas en la proposición anterior, se llama una familia conectificadora de X.

Corolario 1.20. Sea $X$ un espacio de Tychonoff numerable sin puntos aislados. Supóngase que $X$ tiene una compactificación $c X$ tal que existe una sucesión $\left\{\left(x_{n}, y_{n}\right): n \in \omega\right\}$ con las siguientes propiedades: 
(1) $\left\{x_{n}, y_{n}\right\} \subseteq c X \backslash X$ para toda $n \in \omega$;

(2) $\left\{x_{n}, y_{n}\right\} \cap\left\{x_{m}, y_{m}\right\}=\emptyset$ si $n \neq m$;

(9) Para todo par $(U, V)$ de subconjuntos abiertos no vacíos ajenos de $c X$, existe $n \in \omega$ lal que $x_{n} \in U$ y $y_{n} \in V$.

Entonces $X$ es $\omega$-conectificable.

Demostración: Ponemos $F_{n}=\left\{x_{n}, y_{n}\right\}$ y aplicamos la Proposición 1.19.

Definición: La sucesión $F_{n}=\left\{x_{n}, y_{n}\right\}$ con las propiedades enunciadas en el Corolario 1.20 se llama una sucesión conectificadora fuerte para $X$.

En los siguientes dos lemas, las conectificaciones requeridas se obtienen al construir sucesiones conectificadoras fuertes.

Lema 1.21 (BL). Sea $X$ un espacio numerable con $w(X)<\mathfrak{c}$ tal que $X$ es denso en un espacio regular $\left(Z, \tau^{*}\right)$, siendo $Z \backslash X$ separable $y$ denso en $Z$. Entonces X tiene una sucesión conectificadora fuerte (y por lo tanto es $\omega$-conectificable).

Demostración: Dado que $Z \backslash X$ es separable, existe $D$ subconjuto denso y numerable en $Z \backslash X$. Como $Z \backslash X$ es denso en $Z$, se sigue que $D$ es denso en $Z$. Puesto que $X$ es denso en $Z$, por el Lema 1.16 para cada $x \in X$ tenemos $\chi(x, Z)=\chi(x, X) \leq w(X)<c$. Dado que $D$ es denso en $Z$ y numerable, el Lema de Booth implica que para cada $x \in X$ existe una sucesión $S_{x} \subseteq$ $D$ la cual converge a $x[16]$. Sea $\left\{x_{n}: n \in \omega\right\}$ cualquier enumeración de $X$. Entonces existe un mapeo sobreyectivo $\varphi: \omega \backslash\{0\} \rightarrow \omega \times \omega$ tal que $\varphi^{-1}(k, l)$ es infinito para cada $(k, l) \in \omega \times \omega$. Sea $F_{0}$ cualquier subconjunto de $Z \backslash X$ tal que $\left|F_{0}\right|=2$. Supóngase que para algún $n>0$ tenemos definidos subconjuntos $F_{m} \subseteq Z \backslash X$ tales que $F_{k} \cap F_{m}=\emptyset$ si $k \neq m$ y $\left|F_{m}\right|=2$ para todo $m<n$. Si $\varphi(n)=(k, l)$ escogemos parejas de puntos $y_{n} \in S_{x_{k}} \backslash \cup_{m<n} F_{m}$ y $z_{n} \in S_{x_{l}} \backslash \cup_{m<n} F_{m}$ con $y_{n} \neq z_{n}$ y ponemos $F_{n}=\left\{y_{n}, z_{n}\right\}$. Con lo anterior, la construcción inductiva terminó.

Demostremos que $F=\left\{F_{n}: n \in \omega\right\}$ es una familia conectificadora fuerte para $X$. Para $V$ y $U \in T(\beta X)$ escogemos $x_{k} \in X \cap U$ y $x_{l} \in X \cap V$. 
Por las condiciones impuestas a $\varphi$, el conjunto $M=\varphi^{-1}(k, l)$ es infinito y $F_{n} \cap S_{x_{k}} \neq \emptyset \neq F_{n} \cap S_{x_{l}}$ para cada $n \in M$. Más aún, ambos conjuntos $S_{x_{k}} \backslash U$ y $S_{x_{l}} \backslash V$ son finitos puesto que $S_{x_{k}} \rightarrow x_{k}$ y $S_{x_{l}} \rightarrow x_{l}$ mientras que los elementos de la familia infinita $\left\{F_{n}: n \in M\right\}$ son mutuamente ajenos; por lo tanto existe un $n \in M$ tal que $F_{n} \cap U \neq \emptyset \neq F_{n} \cap V$.

Lema $1.22(\mathrm{BL})$. Sea $(X, \tau)$ un espacio numerable de Tychonoff denso en sí mismo, con $\pi w(X)<\mathfrak{c}$. Entonces, $X$ es $\omega$-conectificable.

Demostración: Sea $\gamma$ una $\pi$-base para $X$ tal que $|\gamma|<\mathfrak{c}$. Por el Lema 1.15, existe una topología $\tau^{*}$ de $X$ tal que $\left(X, \tau^{*}\right)$ es de Tychonoff con $\gamma \subseteq \tau^{*} \subseteq \tau$ y $\left|\tau^{*}\right| \leq|\gamma|<$ c. Sea $Y=\left(X, \tau^{*}\right)$ y $i: X \rightarrow Y$ el mapeo identidad de $X$ sobre $Y$. Tomemos $f: \beta X \rightarrow \beta Y$ la extensión continua de $i$. Por el Lema 1.21 existe una familia conectificadora fuerte $\mathcal{F}_{Y}$ para $Y$ con $\cup \mathcal{F}_{Y} \subseteq \beta Y \backslash Y$. Sea

$$
\mathcal{F}_{X}=\left\{f^{-1}(F): F \in \mathcal{F}_{Y}\right\} .
$$

Afirmamos que $\mathcal{F}_{X}$ es una familia conectificadora fuerte para $X$. En efecto, dado que $f$ es una función continua y $\beta X, \beta Y$ son compactos, entonces $\mathcal{F}_{X}$ es una familia de subconjuntos de $\beta X \backslash X$ compactos y mutuamente ajenos. Además, si $U$ es un subconjunto propio abierto-cerrado de $\beta X$ y $V=\beta X \backslash U$, entonces existen $U_{1}$ y $V_{1} \in \gamma$ tales que $U_{1} \subseteq U$ y $V_{1} \subseteq V$. Puesto que $\gamma \subset \tau^{*}$, $i\left(U_{1}\right)$ y $i\left(V_{1}\right)$ son abiertos en $Y$ y por lo tanto sus cerraduras en $\beta Y$ tienen interior no vacío. Así que existe $F \in \mathcal{F}_{Y}$ el cual intersecta ambos interiores y por lo tanto $f^{-1}(F) \cap U \neq \emptyset \neq f^{-1}(F) \cap V$. De lo anterior se sigue que $\mathcal{F}_{X}$ es una familia conectificadora fuerte para el espacio $X$.

Lema 1.23 (BL). Sea $(X, \tau)$ un espacio de Hausdorff numerable y denso en sí mismo con $w(X)<\mathfrak{c}$. Entonces $X$ tiene un subespacio denso regular.

Demostración: Puesto que $w(X)<\mathfrak{c}$, existe una base $\mathcal{B}$ para $X$ con $|\mathcal{B}|<\mathfrak{c}$. Sea $\xi$ la familia de fronteras de elementos de $\mathcal{B}$; entonces para cualquier subfamilia finita $\gamma$ de $\xi$ tenemos $|B \backslash \cup \gamma| \geq \omega$ para todo $B \in \mathcal{B}$, puesto que la unión finita de conjunto densos en ninguna parte es denso en ninguna parte. Así que aplicando el Lema de Booth existe $Y \subseteq X$ tal que $Y \cap B$ es infinito para todo $B \in \mathcal{B}$ y $Y \cap C$ es finito para todo $C \in \xi$. La primera condición 
implica que $Y$ es denso en $X$, mientras que la segunda condición garantiza que existe una base $\mu$ de $Y$ tal que los elementos de esta base tienen fronteras finitas. Sean $y \in Y$ y $U \in T(y, Y)$. Existe $W \in \mu$ tal que $y \in W \subseteq U$. Como $Y$ es de Hausdorff y $W$ tiene frontera finita, existe $V \in T(y, Y)$ tal que $V$ separa a $y$ de la frontera de $W$. Así que $y \in V \cap W \subseteq c_{Y}(V \cap W) \subseteq U$ y por lo tanto $Y$ es regular.

Definición: Sea $X$ un espacio de Tychonoff; se dice que $X$ es $\check{C}$ ech-completo si $X$ es un $G_{\delta}$ en $\beta X$.

Finalmente llegamos a nuestro teorema principal, la generalización del Corolario 1.12.

La conectificación del espacio $X$ en el siguiente teorema se obtiene agregando puntos que no pertenecen a $X$ y definiendo las vecindades de estos puntos en términos de abiertos en $X$ que están asociados a los elementos de un filtro abierto en un subespacio $Y$ denso en $X$.

Teorema 1.24 (BL). Sea $(X, \tau)$ un espacio de Hausdorff, numerable y denso en si mismo con $\pi w(X)<\mathfrak{c}$. Entonces $X$ es $\omega$-conectificable.

Demostración: Dado que $\pi w(X)<\mathfrak{c}$, existe una $\pi$-base $\gamma$ para $X$ con $|\gamma|-\lambda<$ c. $X$ es de Hausdorff y por lo tanto, para todos $x, y \in X$ con $x \neq y$ existe $U_{x, y} \in \tau$ tal que $x \in U_{x, y}$ y $y \notin c l_{X}\left(U_{x, y}\right)$. Sea $\mathcal{B}=\left\{U_{x, y}: x, y \in\right.$ $X\} \cup\left\{X \backslash c l_{X}\left(U_{x, y}\right): x, y \in X\right\}$, se observa que $\mathcal{B}$ es una familia numerable de abiertos que separa puntos de $X$. Sea $\tau_{1}$ la topología en $X$ generada por $\gamma \cup \mathcal{B}$. Por la forma en que se definió $\gamma$ y $\mathcal{B}$, se tiene que $\left(X, \tau_{1}\right)$ es de Hausdorff, $w\left(X, \tau_{1}\right) \leq \lambda$ y $\gamma \subset \tau_{1}$. Así que por el Lema 1.23 existe un subespacio $Y$ de $\left(X, \tau_{1}\right)$ tal que $Y$ es regular y denso en $X$. Sea $x \in X \backslash Y$, definimos $\mathcal{J}_{x}=\{U \cap Y: U \in T(x, X)\}, F_{x}=\cap\left\{c l_{\beta Y}(A): A \in \mathcal{J}_{x}\right\}$ y $H=\cup\left\{F_{x}: x \in X \backslash Y\right\}$.

Veamos que $H \subset \beta Y \backslash Y$. Basta ver que $F_{x} \subset \beta Y \backslash Y$ para todo $x \in X \backslash Y$. Supóngase que $F_{x} \not \subset \beta Y \backslash Y$, entonces existe $y \in F_{x} \cap Y$, es decir

$$
y \in \operatorname{cl}_{\beta Y}(A) \cap Y=c_{Y}(A) \cap Y \quad \text { para todo } A \in \mathcal{J}_{x} .
$$


Por lo tanto $y \in c_{X}(U \cap Y)$ para cada $U \in T(x, X)$, lo cual implica que $x=y$. Esto contradice la elección del punto $x \in X \backslash Y$.

Sea $Z=\beta Y \backslash H$. El espacio $Z$ es Čech-completo ya que $Z=\cap\left\{\beta Y \backslash F_{x}\right.$ : $x \in X \backslash Y\}$, donde $F_{x}$ es cerrado y $X \backslash Y$ es numerable. Por otro lado, afirmamos que $Z \backslash Y$ es separable. Para establecer este hecho, notamos que $Y$ es un espacio regular numerable no disperso con $w(Y) \leq w(X)<\mathfrak{c}$. Entonces por el teorema en [5] $Y$ contiene una copia $P$ de los racionales y $P_{1}=c l_{Z} P$ es Cech-completo; en consecuencia $P_{1} \backslash P$ es denso en $P_{1}$. Por otro lado, si $x \in P$ entonces $\chi\left(x, P_{1}\right)=\chi(x, P)=\omega \mathrm{y}$ por lo tanto para toda $x \in P$ existe una sucesión $S_{x} \subset P_{1} \backslash P$ que converge a $x$. Sea $A_{P}=\cup\left\{S_{x}: x \in P\right\}$. Entonces $A_{P}$ es denso y numerable en $P_{1} \backslash P$, es decir $P_{1} \backslash P$ es separable. Ahora, sea $\mu$ una familia maximal de subespacios mutuamente ajenos de $Y$ homeomorfos a los racionales. Igual que en el Lema 1.18 se tiene que $\cup \mu$ es denso en $Y$. Para cada $P \in \mu$ escogemos un denso numerable $A_{P} \subset \operatorname{cl}_{Z} P \backslash P$ y ponemos $A=\cup\left\{A_{p}: P \in \mu\right\} \subset Z \backslash Y$; entonces $A$ es denso numerable en $Z \backslash Y$, es decir $Z \backslash Y$ es separable.

Ahora por el Lema 1.21 existe una familia numerable conectificadora fuerte para $Y$, digamos $\left\{P_{n}: n \in \omega\right\}$, donde $P_{n}=\left\{x_{n}, y_{n}\right\}$ y $x_{n}, y_{n} \in Z \backslash Y$. Se procederá a construir una conectificación para el espacio $X$.

Sea $\mathcal{U}_{n}=\left\{U \cap Y: U \in T(\beta Y), P_{n} \subset U\right\} ;$ es fácil ver que $\mathcal{U}_{n}$ es un filtro abierto libre en $Y$. Para cada $U \in \mathcal{U}_{n}$ existe $U^{*} \in T(X)$ tal que $U=U^{*} \cap Y$ y definimos $\mathcal{V}_{n}=\left\{U^{*}: U \in \mathcal{U}_{n}\right\}$. Sea $Z^{*}=X \cup\left\{p_{n}: n \in \omega\right\}$ donde $p_{n} \notin X$ para cada $n \in \omega$ y $p_{n} \neq p_{m}$ si $m \neq n$. Definimos una topología en $Z^{*}$ como sigue:

(a) Cada abierto en $X$ es abierto en $Z^{*}$.

(b) Una base local para $p_{n}$ en $Z^{*}$ es la familia $\left\{\left\{p_{n}\right\} \cup U^{*}: U^{*} \in \mathcal{V}_{n}\right\}$.

Veamos que $Z^{*}$ con esta topología es un espacio de Hausdorff. Sea $p_{n} \neq$ $p_{m}$, como $\mathcal{U}_{n} \neq \mathcal{U}_{m}$ entonces existen $U \in \mathcal{U}_{n}$ y $V \in \mathcal{U}_{m}$ tales que $U \cap V=\emptyset$, así que $U^{*} \cap V^{*}=\emptyset$. Se sigue que $\left\{p_{n}\right\} \cup U^{*}$ y $\left\{p_{m}\right\} \cup V^{*}$ son vecindades ajenas de $p_{n}$ y $p_{m}$ respectivamente. Además, $X$ es de Hausdorff y por lo tanto dos puntos distintos de $X$ pueden separarse por vecindades ajenas en $Z^{*}$. Dado que $\beta Y$ también es de Hausdorff, todo punto de $Z^{*} \backslash X$ y todo 
punto de $Y$ pueden separarse por vecindades ajenas. Ahora si $x \in X \backslash Y$ y $p_{n} \in Z^{*} \backslash X$, fijemonos en el filtro abierto $\mathcal{U}_{n}$ generado por $P_{n}$. Puesto que $p_{n} \in Z \subset \beta Y \backslash F_{x}$ tenemos que $P_{n} \cap F_{x}=\emptyset$ y existen $U \in T(x, X)$ y $V \in T\left(P_{n}, \beta Y\right)$ tales que $(U \cap Y) \cap(V \cap Y)=\emptyset$. Sea $V^{*}$ una vecindad de $p_{n}$ con $V^{*} \cap Y=V$. Entonces $U$ y $V^{*}$ son vecindades ajenas de $x$ y $p_{n}$ respectivamente. Por lo tanto $Z^{*}$ es un espacio de Hausdorff.

Veamos que $Z^{*}$ es conexo. Si $U$ es un subconjunto abierto-cerrado no trivial de $Z^{*}$, entonces existen conjuntos no vacíos $V$ y $W \in \gamma$ tales que $V \subset U$ y $W \subset X \backslash U$. De modo que $V \cap Y$ y $W \cap Y$ son subconjuntos abiertos y ajenos no vacíos de $Y$ y por lo tanto existe $n \in \omega$ tal que todo elemento de $\mathcal{U}_{n}$ intersecta a $V$ y $W$. De aqui que $p_{n} \in \operatorname{cl}_{Z^{*}}(U \cap X) \cap c_{Z^{*}}(X \backslash U)$, siendo esto una contradicción. Se sigue que $Z^{*}$ es conexo.

Finalmente $X$ es denso en $Z^{*}$ por construcción. Por lo tanto $X$ es $\omega$ conectificable. 


\section{CAPÍTULO 2}

\section{Conectificación de espacios de Hausdorff y espacios de Tychonoff.}

Condiciones necesarias y suficientes para que un espacio de Hausdorff tenga una conectificación de Hausdorff no son conocidas, sin embargo condiciones suficientes aparecen en [19] y [12]. En [19] se observa que todo espacio de Hausdorff que tiene una conectificación de Hausdorff, no contiene subconjuntos abierto-cerrados no vacíos que sean $H$-cerrados, pero se observa ahí que esta condición no es suficiente.

En este Capítulo, se analizan los resultados descritos en [19] y en [12], referentes a espacios de Hausdorff. En particular se dan dos demostraciones distintas de que la línea de Sorgenfrey es conectificable. Se obtienen también dos condiciones suficientes para que un espacio metrizable tenga una conectificación de Hausdorff y se analizan las hipótesis requeridas para que un espacio cuasirregular numerable y un espacio de Urysohn numerable tengan conectificaciónes de Hausdorff; lo mismo se hace para espacios casi realcompactos. En [17] los espacios considerados son de Tychonoff. Se obtienen condiciones necesarias para que un espacio de Tychonoff y un espacio $n$ realcompacto respectivamente tengan una conectificación de Hausdorff. Se establecen condiciones necesarias y suficientes para que un espacio perfectamente normal tenga una conectificación de Hausdorff.

El primer lema nos permite obtener, de una familia localmente finita, una familia que también es localmente finita pero ajena dos a dos. Dicho lema será usado en el Teorema 2.2, en la Proposición 2.11 y en el Teorema 2.16.

Lema 2.1. Sea $X$ un espacio de Hausdorff sin puntos aislados. Si $\mathcal{B}=$ $\left\{B_{\alpha}: \alpha \in \kappa\right\}$ es una familia localmente finita de subconjuntos abiertos no vacíos de $X$, entonces existe una familia (localmente finita) $\left\{C_{\alpha}: \alpha \in \kappa\right\}$ de subconjuntos abiertos no vacios y ajenos dos a dos tales que $C_{\alpha} \subset B_{\alpha}$ para todo $\alpha \in \kappa$.

Demostración: Como $\mathcal{B}$ es una familia localmente finita de abiertos, para cada $\alpha \in \kappa$ existe $D_{\alpha} \subset B_{\alpha}$ tal que $D_{\alpha}$ intersecta a lo más un número finito de elementos de $\mathcal{B}$. Definimos conjuntos $D_{\alpha}^{*}$ como sigue: 
Introducimos un buen orden en $\kappa^{2}$ y entonces en el paso $(\alpha, \beta)$ ocurren dos casos:

i) Si $D_{\alpha} \cap D_{\beta}=\emptyset$ entonces se definen $D_{\alpha}^{*}=D_{\alpha}$ y $D_{\beta}^{*}=D_{\beta}$.

ii) $D_{\alpha} \cap D_{\beta} \neq \emptyset$; como $D_{\alpha} \cap D_{\beta}$ es un abierto en un espacio $T_{1}$ sin puntos aislados, el Lema 1.2 implica que $D_{\alpha} \cap D_{\beta}$ es un conjunto infinito. Sean $x, y \in D_{\alpha} \cap D_{\beta}$ donde $x \neq y$, siendo $X$ un espacio de Hausdorff, existen abiertos ajenos $V$ y $U$ tales que $x \in V$ y $y \in U$.

Hagamos;

$$
D_{\alpha}^{*}=D_{\alpha} \cap V \quad \text { y } \quad D_{\beta}^{*}=D_{\beta} \cap U .
$$

Puesto que $D_{\alpha} \cap D_{\beta} \neq \emptyset$ ocurre para un número finito de $\beta$ solamente, entonces el proceso anterior se lleva a cabo un número finito de veces obteniéndose al final un elemento $C_{\alpha}$. Así que obtenemos una familia localmente finita $\left\{C_{\alpha}: \alpha \in \kappa\right\}$ de subconjuntos abiertos no vacíos y ajenos dos a dos tales que $C_{\alpha} \subseteq B_{\alpha}$ para cada $\alpha<\kappa$.

Obsérvese que, por construcción, cada elemento de $\left\{C_{\alpha}: \alpha \in \kappa\right\}$ intersecta un número finito de elementos de $\mathcal{B}$.

El siguiente resultado, que aparece en [19], da condiciones suficientes para que un espacio de Hausdorff tenga una conectificación de Hausdorff. La conectificación se construye con puntos de $\omega$, siendo las vecindades de estos puntos determinados por ciertos ultrafiltros abiertos libres en $X$.

Teorema 2.2. Sea $X$ un espacio de Hausdorff con una $\pi$-base $\sigma$-discreta $y$ tal que cada subconjunto abierto no vacio de $X$ pertenece a un ultrafiltro abierto libre. Entonces $X$ tiene una conectificación de Hausdorff.

Demostración: Sea $\mathcal{B}=\cup\left\{\mathcal{C}_{n}: n \in \omega\right\}$ una $\pi$-base de $X$ tal que cada $\mathcal{C}_{n}$ es una familia discreta. Definimos $\mathcal{B}_{n}=\cup\left\{\mathcal{C}_{k}: k \leq n\right\}$ y entonces $\mathcal{B}_{n} \subset \mathcal{B}_{n+1}$. Por inducción, escogeremos ultrafiltros abiertos libres $\left\{\mathcal{U}_{\mathcal{B}}^{i}: B \in \mathcal{B}_{i}\right.$ y $\left.i \in \omega\right\}$ como sigue.

Para $i=1$ y $B \in \mathcal{B}_{1}$, sea $\mathcal{U}_{B}^{1}$ un ultrafiltro abierto libre tal que $B \in \mathcal{U}_{B}^{1}$. Supónganse que para $i<j$ hemos escogido ultrafiltros abiertos libres $\left\{\mathcal{U}_{B}^{i}\right.$ : 
$\left.B \in \mathcal{B}_{i}, i<j\right\}$ tales que para cada $B \in \mathcal{B}_{i}, B \in \mathcal{U}_{B}^{i}$. Dado un $x \in X$, como $\mathcal{C}_{i}$ es discreta para todo $i<j$, existe un abierto $V$ tal que $x \in V$ y $V$ intersecta a lo más $j$ elementos de $\mathcal{B}_{j}$. Así, $\mathcal{B}_{j}$ es una familia localmente finita de abiertos y por el Lema 2.1 existe una familia $\left\{C(B): B \in \mathcal{B}_{j}\right\}$ localmente finita de abiertos no vacíos ajenos dos a dos tal que $C(B) \subset B$ para todo $B \in \mathcal{B}_{j}$. Escogemos $j^{2}+1$ subconjuntos $\left\{C_{B}^{k}: k \leq j^{2}+1\right\}$ de $C(B)$, abiertos no vacíos y ajenos dos a dos (esto es posible por ser $C(B)$ un abierto no vacío en un espacio $T_{2}$ sin puntos aislados). Sea $H_{k, j}=\cup\left\{C_{B}^{k}: B \in \mathcal{B}_{j}\right\}$. Se tiene que $\left\{H_{k, j}: k \leq j^{2}+1\right\}$ es una familia de conjuntos abiertos. Para cada $i<j$ y cada $B \in \mathcal{B}_{i}$, a lo más uno de los conjuntos $H_{k, j}$, es un elemento de $\mathcal{U}_{B}^{i}$. Si un tal $k$ existe hacemos $k(i, B)=k$, si no, escogemos $k(i, B)$ de forma arbitraria. Además, para cada $i<j$ y cada $B \in \mathcal{B}_{i}$ sean:

$$
\begin{aligned}
H(i, B) & =H_{k(i, B)} \cap \cup\left\{C\left(B^{*}\right): B^{*} \in \mathcal{B}_{j} \quad \text { y } \quad C\left(B^{*}\right) \cap B \neq \emptyset\right\}, \\
H(i) & =\cup\left\{H(i, B): B \in \mathcal{B}_{i}\right\} \quad \text { y } \quad H=\cup\{H(i): i<j\} .
\end{aligned}
$$

Se afirma que para cada $B \in \mathcal{B}_{j}, C(B) \backslash H$ contiene algún $C_{B}=C_{B}^{k}$. Para demostrar esto, sea $B \in \mathcal{B}_{j}$ un elemento fijo; entonces:

$$
\begin{gathered}
H\left(k, B^{* *}\right)=\cup\left\{C_{B^{*}}^{k\left(i, B^{* *}\right)}: B^{*} \in \mathcal{B}_{j} \quad \text { y } \quad C\left(B^{*}\right) \cap B^{* *} \neq \emptyset\right\}, \\
\text { donde } i<j \quad \text { y } \quad B^{* *} \in \mathcal{B}_{i} .
\end{gathered}
$$

Así que,

$$
\begin{aligned}
H= & \cup\left\{C_{B^{*}}^{k\left(i, B^{* *}\right)}: B^{*} \in \mathcal{B}_{j} \text { y } C\left(B^{*}\right) \cap B^{* *} \neq \emptyset, i<j, B^{* *} \in \mathcal{B}_{i}\right\} \text { y } \\
& C(B) \backslash H=C(B) \backslash \cup\left\{C_{B}^{k\left(i, B^{* *}\right)}: C(B) \cap B^{* *} \neq \emptyset, i<j, B^{* *} \in \mathcal{B}_{i}\right\} \supset \\
& \cup\left\{C_{B}^{k}: k \leq j^{2}+1\right\} \backslash \cup\left\{C_{B}^{k\left(i, B^{* *}\right)}: C(B) \cap B^{* *} \neq \emptyset, i<j, B^{* *} \in \mathcal{B}_{i}\right\} .
\end{aligned}
$$

Pero para cada $i$, el conjunto $C(B)$ intersecta a lo más $j$ elementos de $\mathcal{B}_{i}$ y por lo tanto existen a lo más $j^{2}$ distintos $k\left(i, B^{* *}\right)$; así que $C(B) \backslash H$ contiene al menos uno de los $C_{B}^{k}$, digamos $C_{B}$.

Ahora para $B \in \mathcal{B}_{j}$ tomemos cualquier ultrafiltro libre $\mathcal{U}_{B}^{j}$ tal que $C_{B} \in$ $\mathcal{U}_{B}^{j}$; con esto la construcción inductiva terminó.

Sea $Y=X \cup \omega$, introducimos una topología en $Y$ como sigue:

i) $V$ es abierto en $Y$ si $V$ es abierto en $X$; 
ii) Tomaremos $\{n\} \cup\left[\cup\left\{U_{B} \in \mathcal{U}_{B}^{n}: B \in \mathcal{B}_{n}\right\}\right]$, como las vecindades en $Y$ de $n \in \omega$.

Veamos que el espacio $Y$ con esta topología es de Hausdorff. Por ser $X$ de Hausdorff, basta separar $i$ de $j$ donde $i<j$. Por construcción, para cada $B \in \mathcal{B}_{j}$ existe $k \leq j^{2}+1$ tal que $C_{B}^{k} \in \mathcal{U}_{B}^{j}$ y donde $C_{B}^{k} \subset C(B) \backslash H$. Para cada $B^{*} \in \mathcal{B}_{i}$ sea $k^{*}=k\left(i, B^{*}\right)$ de ser posible haciendo que se cumpla $H_{k^{*}} \in \mathcal{U}_{B^{*}}^{i}$. Tomemos $D_{B^{*}}^{i} \in \mathcal{U}_{B^{*}}^{i}$, así que $D_{B^{*}}^{i} \cap H_{k^{* *}}=\emptyset$ para todo $k^{*} \neq k^{* *}$ (o para todo $k^{* *}$, si $k^{*}$ no existe). Se afirma que $C_{B}^{k} \cap\left(D_{B^{*}}^{i} \cap B^{*}\right)=\emptyset$. En efecto, tenemos dos casos a considerar:

i) Si $k \neq k^{*}$ o si no existe $k^{*}$, entonces

$$
C_{B}^{k} \cap\left(D_{B^{*}}^{i} \cap B^{*}\right) \subset H_{k} \cap D_{B^{*}}^{i}=\emptyset .
$$

ii) Si $k=k^{*}$, afirmamos que $C(B) \cap B^{*}=\emptyset$, pues de lo contrario se podría tener $C_{B}^{k}=C_{B}^{k^{*}} \subset C(B)$ lo cual no es posible.

Del párrafo anterior se tiene que $\{i\} \cup\left\{D_{B^{*}}^{i} \cap B^{*}: B^{*} \in \mathcal{B}_{i}\right\}$ y $\{j\} \cup$ $\left[\cup\left\{C_{B}^{k}: B \in \mathcal{B}_{j}\right\}\right]$ son vecindades ajenas de $i$ y $j$ respectivamente.

Se sigue que $Y$ es de Hausdorff.

Por construcción $X$ es denso en $Y$. Veamos que $Y$ es conexo. Supongamos, al contrario, que $Y$ es disconexo; entonces existe un abierto-cerrado $K^{\prime} \subset Y$ tal que $K^{\prime} \neq \emptyset$ y $K^{\prime} \neq Y$. Sea $K=X \cap K^{\prime}$. Como $\mathcal{B}_{i} \subset \mathcal{B}_{i+1}$ para todo $i \in \omega$, existen $j \in \omega$ y $B, B^{*} \in \mathcal{B}_{j}$ tales que $B \in K$ y $B^{*} \in X \backslash K$. Así que, $i \in c_{Y} K \cap c_{Y}(X \backslash K)$. Por lo tanto, $K^{\prime}$ no es abierto-cerrado en $Y$.

De todo lo anterior se sigue que $Y$ es una conectificación de Hausdorff para X.

Del teorema anterior se concluyen cuatro resultados importantes, pero para su justificación necesitaremos un lema y unas definiciones.

Una hipótesis, que se debe de satisfacer para aplicar el Teorema 2.2 es que cada subconjunto abierto no vacío de $X$, pertenezca a un ultrafiltro abierto 
libre en $X$, para ello basta que el espacio sea regular y que no tenga subconjuntos compactos con interior no vacío, tal como se indica en el siguiente lema.

Lema 2.3. Sea $X$ un espacio topológico lal que $X$ es regular y no es localmente compacto en ningún punto, entonces cada abierto en $X$ pertenece a un ultrafiltro abierto libre.

Demostración: Ningún punto de $X$ tiene una vecindad compacta y por lo tanto ningún abierto tiene cerradura compacta. Puesto que $X$ es regular, ningún abierto tiene cerradura $H$-cerrada y por lo tanto, cada abierto en $X$ pertenece a un ultrafiltro abierto libre.

\section{Definiciones:}

a) Un espacio X se llama fuertemente Hausdorff por colecciones (s.c.w.H), si para cada subespacio cerrado $\left\{x_{\alpha}: \alpha \in I\right\}$ discreto en $X$, existe una colección discreta $\left\{V_{\alpha}: \alpha \in I\right\} \subseteq T(X)$ tal que $x_{\alpha} \in V_{\alpha}$ para cada $\alpha \in I$.

b) Sea $A \subseteq X$, se dice que $A$ es fuertemente $\sigma$-discreto si $A$ es una unión numerable de cerrados discretos.

Corolario 2.4. Si $X$ es un espacio completamente regular, que en ningún punlo es localmente compacto, siendo primero numerable y s.c.w.H, con un subconjunto denso fuertemente $\sigma$-discreto, entonces $X$ tiene una conectificación de Hausdorff.

Demostración: Sea $D$ un subconjunto denso y fuertemente $\sigma$-discreto, digamos $D=\cup\left\{C_{k}: k \in \omega\right\}$ y donde $C_{k}=\left\{x_{k \alpha}: \alpha \in I_{k}\right\}$ es un conjunto discreto y cerrado. Ahora $X$ es s.c.w.H, por lo tanto para todo $k \in \omega$ existe una familia discreta $\mathcal{F}_{k}=\left\{V_{k \alpha}: \alpha \in I_{k}\right\}$ de abiertos tales que $x_{k \alpha} \in V_{k \alpha}$ para todo $\alpha \in I_{k}$. Como $X$ es primero numerable, es posible escoger una base local de $x_{k \alpha},\left\{U_{k \alpha}^{i}: i \in \omega\right\}$ tal que $x_{k \alpha} \in U_{k \alpha}^{i+1} \subseteq U_{k \alpha}^{i} \subseteq V_{k \alpha}$. Sea $\mathcal{C}_{k}^{i}=\left\{U_{k \alpha}^{i}: \alpha \in I_{k}\right\}$ y $\mathcal{D}=\bigcup\left\{\mathcal{C}_{k}^{i}: k \in \omega\right\}$. 
Veamos que $\mathcal{D}$ es una $\pi$-base para $X$. Sea $V \in T(X)$, entonces $V \cap D \neq \emptyset$, es decir existen $k \in \omega$ y $\alpha \in I_{k}$ tales que $x_{k \alpha} \in V \cap D \subset V$ y así que existe $i \in \omega$ tal que $x_{k \alpha \alpha} \in U_{k \alpha}^{i} \subseteq V$.

Por otro lado, $\mathcal{C}_{k}^{i}$ es un familia discreta para todos $k, i \in \omega$. En efecto, cada elemento de $\mathcal{C}_{k}^{i}$ es un subconjunto de un solo elemento de $\mathcal{F}_{k}$ y como $\mathcal{F}_{k}$ es una familia discreta se sigue que también lo es $\mathcal{C}_{k}^{i}$.

De lo anterior, $X$ tiene una $\pi$-base $\sigma$-discreta. Además, $X$ no es localmente compacto en ningún punto, así que por el Lema 2.3 cada abierto no vacío pertenece a un ultrafiltro abierto libre.

Por el Teorema 2.2, $X$ tiene una conectificación de Hausdorff.

Corolario 2.5. Si $X$ es un espacio metrizable tal que en ningún punto es localmente compacto, entonces $X$ tiene una conectificación de Hausdorff.

Demostración: Como $X$ es metrizable, entonces $X$ es de Hausdorff y por el Teorema de metrización de Bing [7, Teorema 4.4.8], $X$ tiene una base $\sigma$ discreta la misma que es una $\pi$-base $\sigma$-discreta. Así que $X$ es de Hausdorff con una $\pi$-base $\sigma$-discreta. Además, en ningún punto $X$ es localmente compacto, así que por el Lema 2.3 cada abierto en $X$ pertenece a un ultrafiltro abierto libre.

Se sigue por el Teorema 2.2 que $X$ tiene una conectificación de Hausdorff.

Corolario 2.6. Sea $X$ un espacio de Tychonoff, primero numerable y separable, el cual en ningún punto es localmente compacto. Entonces $X$ tiene una conectificación de Hausdorff.

Demostración: El espacio $X$ es separable por lo tanto existe $D=\left\{x_{k}\right.$ : $k \in \omega\}$ un subconjunto denso numerable de $X$. Dado que $X$ es primero numerable, para cada $k \in \omega$ sea $\left\{U_{k}^{i}: i \in \omega\right\}$ la base numerable para $x_{k}$. Hagamos $\mathcal{B}=\cup\left\{\left\{U_{k, i}\right\}: k, i \in \omega\right\}$.

Veamos que $\mathcal{B}$ es una $\pi$-base para $X$. Si $V \in T(X)$, entonces $V \cap D \neq \emptyset$, es decir existe $k \in \omega$ tal que $x_{k} \in V \cap D \subseteq V$, así que existe $i \in \omega$ de manera que $x_{k} \in U_{k i} \subseteq V$. 
Por otro lado $\left\{U_{k i}\right\}$ es una familia discreta para cada $k \in \omega$ y cada $i \in \omega$. Por lo tanto $\mathcal{B}$ es una $\pi$-base $\sigma$-discreta para $X$.

Finalmente por el Lema 2.3, cada abierto no vacío pertenece a un ultrafiltro abierto libre. Se sigue por el Teorema 2.2 que X tiene una conectificación de Hausdorff.

El siguiente resultado fue demostrado por Emeryk y Kulpa en [6].

Corolario 2.7. La línea de Sorgenfrey tiene una conectificación de Hausdorff.

Demostración: La línea de Sorgenfrey es un espacio de Tychonoff, primero numerable y separable, el cual en ningún punto es localmente compacto. Por lo tanto del Corolario 2.6 se concluye que la línea de Sorgenfrey tiene una conectificación de Hausdorff.

Definición: Sea $X$ un espacio de Hausdorff. Dos filtros abiertos $\alpha$ y $\beta$ en $X$, son Hausdorff separados, si existen $U \in \alpha$ y $V \in \beta$ tales que $U \cap V=\emptyset$.

Lema 2.8. Sean $X$ un espacio de Hausdorff y $\mathcal{E}$ un conjunto de filtros abiertos libres en $X$. Sea $Y=X \cup \mathcal{E}$, entonces $\{S \subseteq Y: S \cap \mathcal{E} \neq \emptyset$ y si $\alpha \in S \cap \mathcal{E}$ entonces $S \cap X \in \alpha\} \cup T(X)$ es una topología sobre $Y$, denominada la topología simple y denotada por $\tau_{\mathcal{E}}$. Además, $X$ es denso en $\left(Y, \tau_{\mathcal{E}}\right)$.

\section{Demostración:}

i) $\emptyset \in T(X) \subset \tau_{\mathcal{E}}$, por lo tanto $\emptyset \in \tau_{\mathcal{E}}$. Además, puesto que todo filtro $\alpha$ en $X$ contiene a $X$, sí $\alpha \in Y \cap \mathcal{E}$, entonces se tiene que $Y \cap X=X \in \alpha$, es decir $Y \in \tau_{\mathcal{E}}$.

ii) Sean $A, B \in \tau_{\mathcal{E}}$ existen cuatro posibilidades.

a) Si $A, B \in T(X)$, entonces $A \cap B \in T(X) \subseteq \tau_{\mathcal{E}}$.

b) Si $A, B \notin T(X)$, entonces $A \cap \mathcal{E} \neq \emptyset$ y $B \cap \mathcal{E} \neq \emptyset$. Ahora, si $(A \cap B) \cap \mathcal{E}=(A \cap \mathcal{E}) \cap(B \cap \mathcal{E})=\emptyset$ se sigue que $A \cap B \subseteq X$ y por lo tanto $A \cap B=(A \cap X) \cap(B \cap X)$; pero $A \cap X$ y $B \cap X$ son 
abiertos en $X$ y por lo tanto $A \cap B \in T(X) \subset \tau_{\mathcal{E}}$. Por otro lado, si $(A \cap B) \cap \mathcal{E} \neq \emptyset$ entonces para todo $\alpha \in(A \cap B) \cap \mathcal{E}$ se tiene $\alpha \in A \cap \mathcal{E}$ y $\alpha \in B \cap \mathcal{E}$, por lo tanto $A \cap X \in \alpha$ y $B \cap X \in \alpha$; pero $\alpha$ es un filtro y se sigue que $(A \cap B) \cap X=(A \cap X) \cap(B \cap X) \in \alpha$. Es decir $A \cap B \in \tau_{\mathcal{E}}$.

c) Si $A \in T(X)$ y $B \notin T(X)$, entonces $A$ es abierto en $X$ y $B \cap X$ es abierto en $X$; por lo tanto $A \cap B=A \cap(B \cap X) \in T(X) \subseteq \tau_{\mathcal{E}}$.

d) Si $B \in T(X)$ y $A \notin T(X)$, al igual que en (c) se concluye que $A \cap B \in \tau_{\mathcal{E}}$.

iii) Sea $I$ un conjunto de índices y supongamos que $A_{\lambda} \in \tau_{\mathcal{E}}$ para toda $\lambda \in I$. Si tomamos $A=\cup\left\{A_{\lambda}: \lambda \in I\right\}$; Existen tres posibilidades:

a) $A_{\lambda} \in T(X)$ para toda $\lambda \in I$, entonces $A \in T(X) \subseteq \tau_{\mathcal{E}}$.

b) $A_{\lambda} \notin T(X)$ para toda $\lambda \in I$, entonces $A_{\lambda} \cap \mathcal{E} \neq \emptyset$ y $A_{\lambda} \cap X \neq \emptyset$ para todo $\lambda \in I$, se sigue que $A \cap \mathcal{E} \neq \emptyset$ y $A \cap X \neq \emptyset$ por lo tanto para todo $\alpha \in A \cap \mathcal{E}$ se tiene que $\alpha \in A_{\kappa} \cap \mathcal{E}$ para algún $\kappa \in I$. Pero entonces $A_{\kappa} \cap X \in \alpha$ y como $A_{\kappa} \cap X \subset A \cap X$ se sigue que $A \cap X \in \alpha$ para todo $\alpha \in A \cap \mathcal{E}$. Por lo tanto $A \in \tau_{\mathcal{E}}$.

c) Supóngase que existe $J \subset I, J \neq I$ tal que $A_{\lambda} \notin T(X)$ para toda $\lambda \in J$. Entonces $B=\cup\left\{A_{\lambda}: \lambda \in I \backslash J\right\} \in T(X)$ y $C=$ $\cup\left\{A_{\lambda}: \lambda \in J\right\} \in \tau_{\mathcal{E}} \backslash T(X)$ por los incisos (i) y (ii). Se sigue que $C \cap \mathcal{E} \neq \emptyset$ y por lo tanto $(B \cup C) \cap \mathcal{E} \neq \emptyset$. Sea $\alpha \in(B \cup C) \cap \mathcal{E}$, entonces $\alpha \in C \cap \mathcal{E}$ así que $C \cap X \in \alpha$ de donde $(B \cup C) \in \alpha$ y en consecuencia $A \in \tau_{\mathcal{E}}$.

Se tiene por lo tanto que $\tau_{\mathcal{E}}$ es una topología en $Y$.

Finalmente, veamos que $X$ es denso en $Y$. Sea $A \in \tau_{\mathcal{E}}$. Hay dos posibilidades:

i) Si $A \in T(X)$, entonces $A \cap X \neq \emptyset$.

ii) Si $A \in \tau_{\mathcal{E}} \backslash T(X)$, entonces $A \cap \mathcal{E} \neq \emptyset$; por lo tanto si $\alpha \in A \cap \mathcal{E}$ se tiene que $A \cap X \in \alpha$ y como $\alpha$ es un filtro, entonces $A \cap X \neq \emptyset$.

De lo anterior se tiene que $X$ es denso en $Y$. 
En el lema anterior se tiene que $X$ es denso en $Y$. Para lograr que $Y$ sea un espacio de Hausdorff conexo, basta pedir dos condiciones sobre $\mathcal{E}$ como se indica en el proximo lema. Dicho lema aparece en [12].

Lema 2.9. Sea $X$ un espacio de Hausdorff y $\mathcal{E}$ un conjunto de filtros abiertos libres en X. Supóngase que

a) Cada par de elementos de $\mathcal{E}$ son Hausdorff separados.

b) Si $C$ es un conjunto abierto-cerrado no trivial en $X$, existe $\alpha \in \mathcal{E}$ tal que para todo $U \in \alpha, U \cap C \neq \emptyset \neq U \cap(X \backslash C)$.

Entonces $\left(Y, \tau_{\mathcal{E}}\right)$ es una conectificación de Hausdorff del espacio $X$.

Demostración: Veamos que $Y$ es un espacio conexo. Si no es así, entonces existe un abierto-cerrado $C$ en $Y$ tal que $C$ es no trivial y como $X$ es denso en $Y$, se sigue que $A=C \cap X$ es un abierto-cerrado no trivial en $X$. Así que por el inciso (b) existe un filtro $\alpha \in \mathcal{E}$ tal que $A \cap U \neq \emptyset \mathrm{y}(X \backslash A) \cap U \neq \emptyset$ para todo $U \in \alpha$. Sea $V$ un abierto en $Y$ que contiene a $\alpha$ (por ejemplo, si $U \in \alpha$ entonces podemos tomar $V=U \cup\{\alpha\})$, entonces $V \cap X \in \alpha$. Se sigue que $A \cap(V \cap X) \neq \emptyset \mathrm{y}(X \backslash A) \cap(V \cap X) \neq \emptyset$, es $\operatorname{decir} V \cap A \neq \emptyset \mathrm{y}$ $V \cap(\mathrm{X} \backslash A) \neq \emptyset$. Por lo tanto $V \cap C \neq \emptyset$ y $V \cap(Y \backslash C) \neq \emptyset$ para todo abierto $V$ en $Y$ que contiene a $\alpha$. Así que, $\alpha \in c_{Y} C \cap c l_{Y}(Y \backslash C)=C \cap(Y \backslash C)=\emptyset$ lo cual es una contradicción.

Por otro lado veamos que $Y$ es un espacio de Hausdorff. Como $X$ es de Hausdorff y es abierto en $Y$, basta considerar dos casos:

i) Si $\alpha$ y $\beta$ son dos filtros distintos en $\mathcal{E}$, se tiene por el inciso (a) que existen $U \in \alpha$ y $V \in \beta$ tales que $V \cap U=\emptyset$. Sean $U^{*}=\{\alpha\} \cup U$ y $V^{*}=\{\beta\} \cup V$; entonces $U^{*}$ y $V^{*}$ son abiertos ajenos en $Y$ que contienen a $\alpha$ y $\beta$ respectivamente.

ii) Sean $x \in X$ y $\alpha \in \mathcal{E}$; puesto que $\alpha$ es libre, existe $U \in \alpha$ tal que $x \notin$ $c l(U)$; sea $V$ una vecindad abierta de $x$ tal que $V \cap U=\emptyset$. Definiendo $U^{*}=\{\alpha\} \cup U$, concluimos que $V$ y $U^{*}$ son abiertos ajenos en $Y$ tales que $x \in V$ y $\alpha \in U^{*}$. 
Se sigue que $Y$ es un espacio de Hausdorff. Además, por el Lema 2.8 $X$ es denso en $Y$. Se tiene por lo tanto que $X$ tiene una conectificación de Hausdorff.

Se observa que en las hipótesis del Teorema 2.2 a parte de pedir una $\pi$-base $\sigma$-discreta para $X$, se pide también que todo subconjunto abierto no vacío de $X$ pertenezca a un ultrafiltro abierto libre en $X$. En el siguiente resultado, que es consecuencia del Lema 2.9 , se requiere únicamente que a cada subconjunto $U$ abierto-cerrado no trivial de $X$, se le asigne de forma inyectiva, un ultrafiltro abierto libre que contenga a $U$.

Lema 2.10. Sea $X$ un espacio de Hausdorff. Si existe una función inyectiva $U \rightarrow \mathcal{U}_{U}$ que asigna a cada subconjunto abierto-cerrado no trivial $U \subset X$, un ultrafiltro abierto libre $\mathcal{U}_{U}$ que contiene a $U$, entonces $X$ tiene una conectificación de Hausdorff.

Demostración: Sea, $\mathcal{E}-\left\{\mathcal{F}_{U}=\mathcal{U}_{U} \cap \mathcal{U}_{X \backslash U}: U\right.$ es un subconjunto abiertocerrado no trivial de $X\}$ un conjunto de filtros abiertos libres en $X$. Entonces veamos que se cumplen los condiciones del Lema 2.9:

a) Suponer que $\mathcal{F}_{U}, \mathcal{F}_{V} \in \mathcal{E}$; puesto que $\mathcal{U}_{U}, \mathcal{U}_{X \backslash U}, \mathcal{U}_{V}, \mathcal{U}_{X \backslash V}$ son ultrafiltros abiertos distintos podemos escoger $F_{1} \in \mathcal{U}_{U}, F_{2} \in \mathcal{U}_{X \backslash U}, F_{3} \in \mathcal{U}_{V}$, $F_{4} \in \mathcal{U}_{X \backslash V}$ mutuamente ajenos; entonces $F_{1} \cup F_{2} \in \mathcal{F}_{U}$ y $F_{3} \cup F_{4} \in \mathcal{F}_{V}$ son ajenos.

b) Sea $U \subset X$, un abierto-cerrado no trivial en $X$, entonces $U \in \mathcal{U}_{U}$ y $(X \backslash U) \in \mathcal{U}_{X \backslash U}$. Sea $V \in \mathcal{F}_{U}=\mathcal{U}_{U} \cap \mathcal{U}_{X \backslash U}$. Se tiene entonces que $U, V \in \mathcal{U}_{U}$ y $(X \backslash U), V \in \mathcal{U}_{X \backslash U^{\prime}}$, por lo tanto $U \cap V \neq \emptyset$ y $(X \backslash U) \cap V \neq \emptyset$ para toda $V \in \mathcal{F}_{U}$.

Por el Lema 2.9, X tiene una conectificación de Hausdorff.

Definición: Sea $X$ un espacio topologíco. Definimos $\mathcal{C}(X)$ como la familia de subconjuntos abierto-cerrados no triviales de $X$. 
La siguiente proposición inicia una serie de resultados, que nos permiten concluir cuando un espacio de Hausdorff X tiene una conectificación de Hausdorff, en terminos de la cantidad de subconjuntos abierto-cerrados de $X$.

Proposición 2.11. Un espacio $X$ de Hausdorff liene una conectificación de Hausdorff, si cada subconjunto $U$ abierto-cerrado no trivial de $X$, no es débilmente compacto y $|\mathcal{C}(X)| \leq 2^{\mathfrak{C}}$.

Demostración: Sea $U$ un subconjunto abierto-cerrado no trivial de $X$. Puesto que $U$ no es débilmente compacto, existe una familia localmente finita $\mathcal{F}=\left\{V_{n}: n \in \omega\right\}$ de abiertos no vacíos tales que $V_{n} \subset U$ para cada $n \in \omega$. Tomando en cuenta el Lema 2.1, se ve que podemos asumir que los elementos de $\mathcal{F}$ son mutuamente ajenos. Ahora usando la misma construcción que usamos en el Lema 1.10 , se obtienen $2^{\mathfrak{c}}$ ultrafiltros abiertos libres que contienen a $U$. Sea $\left\{U_{\alpha}: \alpha<2^{\mathfrak{c}}\right\}$ un buen ordenamiento de $\mathcal{C}(X)$ y definimos $\mathcal{U}^{*}\left(U_{\alpha}\right)=\left\{\mathcal{U}: \mathcal{U}\right.$ es un ultrafiltro abierto libre que contiene a $\left.U_{\alpha}\right\}$. Por un proceso inductivo hagamos la siguiente correspondencia: dado $U_{1}$ escogemos cualquier $\mathcal{U}_{1} \in \mathcal{U}^{*}\left(U_{1}\right)$. Supóngase que para $\alpha<\beta$ hemos escogido $\mathcal{U}_{\alpha} \in \mathcal{U}^{*}\left(U_{\alpha}\right)$ de tal manera que $\mathcal{U}_{\alpha} \neq \mathcal{U}_{\gamma}$ si $\alpha>\gamma$. Puesto que $2^{\mathfrak{c}} \leq\left|\mathcal{U}^{*}\left(U_{\beta}\right)\right|$, existe $\mathcal{U}_{\beta} \in \mathcal{U}^{*}\left(U_{\beta}\right) \backslash \cup\left\{\mathcal{U}_{\alpha}: \alpha<\beta\right\}$. Por lo tanto está definida una correspondencia biunivoca $U_{\beta} \rightarrow \mathcal{U}_{\beta}$, así que por el Lema $2.10, X$ tiene una conectificación de Hausdorff.

Corolario 2.12. Un espacio de Hausdorff $X$ tiene una conectificación de Hausdorff, si cada subconjunto abierto-cerrado no trivial de $X$ no es débilmenle compacto y $d(X) \leq \mathfrak{c}$.

Demostración: Sea $D \subseteq X$ tal que $D$ es denso en $X$ y $|D| \leq \mathfrak{c}$. De aquí se sigue que $|\mathcal{P}(D)| \leq 2^{\mathfrak{c}}$. Por otro lado si $U \in \mathcal{C}(X)$ entonces la función $U \rightarrow U \cap D$, es inyectiva. De lo anterior se tiene que

$$
|\mathcal{C}(X)| \leq|\mathcal{C}(D)| \leq|\mathcal{P}(D)| \leq 2^{\mathfrak{C}} .
$$

Además, por hipótesis, ningún elemento de $\mathcal{C}(X)$ es débilmente compacto, así que por la Proposición $2.11 X$ tiene una conectificación de Hausdorff. 
Definición: Un espacio $X$ es casi realcompacto, si para todo $\mathcal{U}$ ultrafiltro abierto libre sobre $X$ existe $\mathcal{G} \subset U$ numerable tal que $\cap\{c l(G): G \in \mathcal{G}\}=\emptyset$.

Corolario 2.13. Sea $X$ un espacio de Hausdorff casi realcompacto. Si ningún subconjunto abierto-cerrado no trivial de $X$ es $H$-cerrado y $|\mathcal{C}(X)| \leq$ $2^{\mathfrak{c}}$, entonces $X$ tiene una conectificación de Hausdorff.

Demostración: Sea $U \in \mathcal{C}(X)$, veamos que $U$ es casi realcompacto. Sea $\mathcal{U}$ un ultrafiltro abierto libre en $X \operatorname{con} U \in \mathcal{U}$. Dado que $X$ es casi realcompacto, existe $\mathcal{G} \subseteq U$ numerable tal que $\cap\{c l(G): G \in \mathcal{G}\}=\emptyset$, así que $\cap\{c l(G \cap U)$ : $G \in \mathcal{G}\}=\emptyset$. Se sigue que $U$ es casi realcompacto. Pero, todo espacio débilmente compacto y casi realcompacto es $H$-cerrado, pues si $U$ no es $H$-cerrado, entonces existe un ultrafiltro abierto libre $\mathcal{U}$ tal que $U \in \mathcal{U}$. Dado que $U$ es casi realcompacto, entonces existe $\left\{G_{n}: n \in \omega\right\} \subset \mathcal{U}$ tal que $\cap\left\{c l_{U}\left(G_{n}\right): n \in \omega\right\}=\emptyset$. Sea $I_{n}=\cap\left\{G_{m}: 1 \leq m \leq n\right\}$, se ticne que $\{H n: n \in \omega\}$ es una familia anidada de abiertos tales que $\cap\left\{c l_{U}\left(H_{n}\right): n \in \omega\right\}=\emptyset$, es decir $U$ no es débilmente compacto y se tiene por lo tanto una contradicción.

Ahora por hipótesis $X$ no tiene subespacios abiertos $H$-cerrados, asi que $U$ no es débilmente compacto. Por otro lado $|\mathcal{C}(X)| \leq 2^{\mathfrak{c}}$ y se sigue de la Proposición 2.11 que $X$ tiene una conectificación de Hausdorff.

Dado que la recta de Sorgenfrey $\mathbb{S}$ es separable, realcompacta (por ser de Lindelöf) y en consecuencia casi realcompacta por [8, Teorema 10] y sin subconjuntos abierto-cerrados $H$-cerrados distintos del vacío, por lo tanto el Corolario 2.13 proporciona otra demostración de que la recta de Sorgenfrey es conectificable. Esta demostración es distinta a la dada en el Corolario 2.7.

La Proposición 2.11 permite conectificar espacios cuasirregulares y espacios de Urysohn, de cardinalidad menor que el continuo.

Definiciones: Sea $X$ un espacio topológico.

a) $X$ es cuasirregular si para todo $U$, abierto no vacío en $X$, existe $V$, abierto no vacío en $X$, tal que $V \subset \mathrm{cl} V \subset U$. 
b) $X$ es de Urysohn si para todo $x, y \in X, x \neq y$ existen $U, V$ abiertos en $X$ tales que $x \in U, y \in V$ y $c l U \cap c l V=\emptyset$.

Proposición 2.14. Sea $X$ un espacio de Hausdorff, denso en sí mismo $y$ cuasirregular tal que $|X|<\mathfrak{c}$, entonces $X$ tiene una conectificación de Hausdorff.

Demostración: Sea $U$ un subconjunto abierto-cerrado no trivial de $X$, se afirma que $U$ no es débilmente compacto. Para demostrar esto supóngase que $U$ es débilmente compacto. Como $X$ es un espacio de Hausdorff y denso en sí mismo se sigue que $U$ es infinito. Sean $x, y \in U$ donde $x \neq y$, entonces existen abiertos ajenos $V_{1}, V_{2}$ tales que $x \in V_{1} \subset U$ y $y \in V_{2} \subset U$. Ahora, puesto que $X$ es cuasirregular, existen abiertos no vacíos $W_{1}$ y $W_{2}$ tales que $W_{1} \subset \operatorname{cl} W_{1} \subset$ $V_{1} \mathrm{y} W_{2} \subset c l W_{2} \subset V_{2}$, se sigue que $c l W_{1}$ y $c l W_{2}$ son cerrados ajenos. Sea $T_{1}=$ $\left\{W_{1}, W_{2}\right\}$. Aplicando el mismo razonamiento para los subconjuntos abiertos $W_{1}$ y $W_{2}$, obtenemos $T_{2}=\left\{c l W_{11}, c l W_{12}, c l W_{21}, c l W_{22}\right\}$ donde; $c l W_{11}$ y $c l W_{12}$ son cerrados ajenos contenidos en $W_{1}$ y $c l W_{21}$ y $c l W_{22}$ son cerrados ajenos contenidos en $W_{2}$. Procediendo de la misma forma, por un proceso inductivo se construye un árbol de Cantor donde cada elemento del $n$-ésimo nivel $T_{n}$ es un cerrado regular y además estos cerrados regulares son ajenos. Por el Teorema 1.6, si $U$ fuera débilmente compacto, la intersección de los elementos de una rama maximal de este árbol tendría que ser no vacía, pues la rama cs una cadena numcrable de cerrados regulares. Puesto que el árbol tiene $c$ ramas, se sigue que $\mathfrak{c} \leq|U|$; pero $|U| \leq|X|<\mathfrak{c}$ por lo tanto tenemos una contradicción. Se sigue que ningún abierto-cerrado no trivial es débilmente compacto.

Además, $|\mathcal{C}(X)| \leq|\mathcal{P}(X)|=2^{|X|} \leq 2^{\mathfrak{C}}$ y se sigue de la Proposición 2.11. que $X$ tiene una conectificación de Hausdorff.

El resultado anterior es básicamente la Proposición 2.15 de [1] salvo que la hipótesis de que el espacio sea de Urysohn es cambiado por espacio cuasirregular. En este caso la demostración de la proposición 2.14 es similar a la demostración de 2.15 de [1]. Observemos que Urysohn y cuasirregular son conceptos independientes pues, existen espacios de Urysohn que no son cuasirregulares e inversamente, por ejemplo; 
Sea $A=\{-1 / n: n \in \omega\} \cup\{0\} \cup\{1 / n: n \in \omega\}$ un conjunto linealmente ordenado con la topología de orden y $Z^{+}$el conjunto de los enteros positivos con la topología discreta. Tomemos $X=A \times Z^{+} \cup\{\alpha,-\alpha\}$ donde todo abierto en la topología producto $A \times Z^{+}$es abierto en $X$ y las vecindades de $\alpha y-\alpha$ son conjuntos de la forma:

$$
\begin{aligned}
& M_{n}^{+}(\alpha)=\{\alpha\} \cup\{(1 / m, j): m<\omega \quad \mathrm{y} \quad n<j\} \quad \mathrm{y} \\
& M_{n}^{-}(-\alpha)=\{-\alpha\} \cup\{(-1 / m, j): m \in \omega \quad \mathrm{y} \quad n<j\}
\end{aligned}
$$

respectivamente.

Entonces cada vecindad básica contiene un punto aislado y por lo tanto $X$ es cuasiregular. Pero $\alpha$ y $-\alpha$ son elementos de $X$ que no se pueden separar por abiertos cuyas cerraduras sean ajenas, es decir, $X$ no es de Urysohn. Por otro lado el espacio numerable, conexo y de Hausdorff construido en [15] es de Urysohn pero no es cuasirregular.

Para establecer notación, reformularemos el Lema 2.1 como sigue:

Si $\mathcal{F}$ es una familia localmente finita de subconjuntos abiertos no vacíos en un espacio de Hausdorff $X$, sin puntos aislados, entonces existe una familia localmente finita $\mathcal{G}(\mathcal{F})=\{G(F): F \in \mathcal{F}\}$ de subconjuntos abiertos no vacíos y ajenos dos a dos tales que $G(F) \subset F$ para cada $F \in \mathcal{F}$.

De la observación, seguida del Lema 2.1, se sigue que cada elemento de $\mathcal{G}(\mathcal{F})$ intersecta un número finito de elementos de $\mathcal{F}$, por lo tanto $|\mathcal{F}|=$ $|\mathcal{G}(\mathcal{F})|$.

En lo que sigue necesitamos el siguiente resultado;

Lema 2.15. $\mid\{C: C$ es un subconjunto propio abierto-cerrado de $M\} \mid \leq \mathfrak{c}$ si $\pi w(M) \leq w$.

Demostración: Sea $\mathcal{B}$ una $\pi$-base de $M$ con $|\mathcal{B}| \leq \omega$. Tomemos $\mathcal{F}_{C}$ una familia maximal de elementos mutuamente ajenos de $\mathcal{B}$ contenidos en $C$. Si $F \in \mathcal{F}_{C}$ entonces, $c l(F) \subset C$ y $\cup F_{C}$ es denso en $C$. Por lo tanto $C=c l\left(\cup F_{C}\right)$. O sea, cada abierto-cerrado estáunicamente determinado por una subfamilia 
de elementos de $\mathcal{B}$. Se sigue que, $\mid\{C: C$ es un subconjunto propio abiertocerrado de $M\}|\leq| P(\mathcal{B}) \mid=2^{|\mathcal{B}|} \leq \mathfrak{c}$.

Se sabe por el Teorema 2.2 que un espacio de Hausdorff con una $\pi$-base $\sigma$-discreta y tal que cada subconjunto abierto no vacío de $X$ pertenece a un ultrafiltro abierto libre, tiene una conectificación de Hausdorff. El siguiente resultado, que aparece en [12] y es consecuencia del Lema 2.9, requiere que el espacio tenga una $\pi$-base $\sigma$-localmente finita además de no poseer subconjuntos abiertos $H$-cerrados y que $\omega_{1} \leq \pi w(X)$. Ambas demostraciones usan la noción de ultrafiltros, pero en el presente resultado necesitaremos ultrafiltros uniformes.

Definición: Una familia $\mathcal{U}$ es un ultrafiltro uniforme en $X$ si para todo $U \in \mathcal{U}$ se tiene que $|U|=|X|$.

Teorema 2.16. Sea $X$ un espacio de Hausdorff, con $\omega_{1} \leq \pi w(X)$ y una $\pi$ base $\sigma$-localmente finita, tal que $X$ no tiene subconjuntos abiertos $H$-cerrados distintos del vacio, entonces $X$ tiene una conectificación de Hausdorff.

Demostración: Dado que $X$ tiene una $\pi$-base $\sigma$-localmente finita $\mathcal{C}=$ $\cup\left\{\mathcal{C}_{n}: n \in \omega\right\}$ donde cada $\mathcal{C}_{n}$ es localmente finita y $\omega_{1} \leq \pi w(X)$, entonces se puede suponer que $\omega_{1} \leq\left|\mathcal{C}_{0}\right|$. Se afirma que existe una $\pi$-base $\mathcal{B}=\cup\left\{\mathcal{B}_{n}: n \in \omega\right\}$ tal que;

(i) Cada $\mathcal{B}_{n}$ es una familia localmente finita de subconjuntos abiertos y ajenos dos a dos, tales que $\omega_{1} \leq\left|\mathcal{B}_{n}\right|$.

(ii) Si $n<m$ y $B \in \mathcal{B}_{n}$, entonces existe $C \in \mathcal{B}_{m}$ tal que $C \subset B$ y $C \neq B$.

(iii) Si $C \in \mathcal{B}_{m}$ y $C \cap B \neq \emptyset$ para algún $B \in \mathcal{B}_{n}$ donde $n \leq m$, entonces $C \subset B$.

Dicha $\pi$-base se obtiene por un proceso inductivo como sigue; dado que $\mathcal{C}_{0}$ es una familia localmente finita, por la reformulación del Lema 2.1, se puede tomar $\mathcal{B}_{0}=\mathcal{G}\left(\mathcal{C}_{0}\right)$; entonces $\mathcal{B}_{0}$ en una familia localmente finita de subconjuntos abiertos ajenos dos a dos; además, por la observación en la reformulación del Lema 2.1 se tiene que $\omega_{1} \leq\left|\mathcal{C}_{0}\right|=\left|\mathcal{G}\left(\mathcal{C}_{0}\right)\right|=\left|\mathcal{B}_{0}\right|$, es decir se cumple (i). 
Supóngase que hemos definido $\mathcal{B}_{k}$, para $k \leq n$, tal que se cumplen (i), (ii) y (iii). Ahora sean;

$$
\mathcal{B}_{n+1}^{* *}=\mathcal{G}\left(\mathcal{B}_{n} \cup \mathcal{C}_{n+1}\right) \quad y
$$

$\mathcal{B}_{n+1}^{*}=\left\{B \cap F: B \in \mathcal{B}_{n} \quad\right.$ y $\left.\quad F \in \mathcal{B}_{n+1}^{* *}\right\} \cup\left\{F \in \mathcal{B}_{n+1}^{* *}: F \cap\left(\cup \mathcal{B}_{n}\right)=\emptyset\right\}$.

Como $X$ es $T_{1} \mathrm{y}$ sin puntos aislados entonces, por el Lema 1.2, los elementos de $\mathcal{B}_{n+1}^{*}$ son abiertos infinitos, así que se puede definir $\mathcal{B}_{n+1}=$ $\left\{D \backslash\{p\}: D \in \mathcal{B}_{n+1}^{*}\right.$ y $\left.p \in D\right\}$. Entonces, $\mathcal{B}_{n+1}$ es una familia localmente finita, pues $\mathcal{C}_{n+1}$ y $\mathcal{B}_{n}$ lo son; además, $\omega_{1} \leq\left|\mathcal{B}_{n}\right| \leq\left|\mathcal{B}_{n+1}^{*}\right|=$ $\left|\mathcal{B}_{n+1}\right|$, por lo tanto se cumple (i). Para probar (ii) y (iii) basta verificar las condiciones para $n$ y $n+1$. Probemos que se cumple (ii); para ello, sea $B \in \mathcal{B}_{n}$, entonces si $F \in \mathcal{B}_{n+1}^{* *}$ se sigue que $(B \cap F) \backslash\{p\} \subset B$ y $(B \cap F) \backslash\{p\} \neq B$ si $p \in B \cap F$. Puesto que $(B \cap F) \backslash\{p\} \in \mathcal{B}_{n+1}$, entonces se cumple (ii). Ahora, si $C \in \mathcal{B}_{n+1}$ y $C \cap B \neq \emptyset$ para algún $B \in \mathcal{B}_{n}$, entonces existe $D \in \mathcal{B}_{n+1}^{*}$, tal que $C=D \backslash\{p\}$ donde $p \in D$. Se sigue que $D \cap B \neq \emptyset$, (ya que $p$ es un punto arbitrario de $D$ ), así que $D \cap\left(\cup \mathcal{B}_{n}\right) \neq \emptyset$, y por lo tanto $D$ es de la forma $B \cap F$ para $F \in \mathcal{B}_{n+1}^{* *}$, lo cual implica que $D \subset B$ y por lo tanto $C \subset B$. Así que se cumple (iii).

Se observa que $\mathcal{B}_{n} \cap \mathcal{B}_{m}=\emptyset$ si $n \neq m$, pues si existe $B \in \mathcal{B}_{n} \cap \mathcal{B}_{m}$, entonces por (ii) si $m>n$ existe $C \subset \mathcal{B}_{m}$ tal que $C \subset B$ y $C \neq B$, pero $B \in \mathcal{B}_{m}$, así que por (i) se tiene que $B$ y $C$ deberían ser ajenos lo cual es imposible. Ahora dado que $X$ es un espacio $T_{1}$ sin puntos aislados, por el Lema 1.2 , se pueden escoger inductivamente puntos $p_{B}, q_{B} \in B$ para cada $B \in \mathcal{B}$ de tal forma que $p_{B}, q_{B}, p_{C}, y q_{C}$ sean cuatro puntos distintos siempre y cuando $B, C \in \mathcal{B}$ y $B \neq C$.

(iv) Si $C \in \mathcal{C}(X)$ y $\omega<\pi w(C)$, entonces existe algún $n \in \omega$ tal que $\omega<\left|\left\{B \in \mathcal{B}_{n}: B \subset C\right\}\right|$. Con este valor fijo de $n$, se sigue de (ii) que todo elemento $B \in \mathcal{B}_{n}$ contiene un elemento de $\mathcal{B}_{m}$ para $1 \leq n \leq m$; por lo tanto $\omega<\left|\left\{B \in \mathcal{B}_{m}: B \subset C\right\}\right|$ para todo $n \leq m$.

Para cada $n \in \omega$, sea $\mathcal{F}_{n}=\left\{U \in T(X):\left|\left\{B \in \mathcal{B}_{n}: p_{B} \notin U\right\}\right|<\omega\right\}$. Se observa que $\mathcal{F}_{n}$ es un filtro abierto en $X$, ya que $\left\{p_{B}: B \in \mathcal{B}_{n}\right\}$ es infinito y por lo tanto, si $U$ y $V \in \mathcal{F}_{n}$ entonces $\left\{B \in \mathcal{B}_{n}: p_{B} \notin U\right\}$ y $\left\{B \in \mathcal{B}_{n}: p_{B} \notin V\right\}$ son finitos, así que $\left\{B \in \mathcal{B}_{n}: p_{B} \notin U \cap V\right\}$ es finito, es decir $U \cap V \in \mathcal{F}_{n}$ y $\emptyset \notin \mathcal{F}_{n}$. 
(v) Se afirma que $\mathcal{F}_{n}$ es un filtro abierto libre, puesto que si $x \in \cap\left\{c l_{X} U\right.$ : $\left.U \in \mathcal{F}_{n}\right\}$ entonces dado que $\mathcal{B}_{n}$ es una familia localmente finita, existe $W \in T(X)$ tal que $x \in W$ y $\left|\left\{B \in \mathcal{B}_{n}: B \cap W \neq \emptyset\right\}\right|<\omega$. Sea $V=\cup \mathcal{B}_{n} \backslash \cup\left\{B \in \mathcal{B}_{n}: B \cap W \neq \emptyset\right\}$, entonces por construcción $V \in \mathcal{F}_{n}$ y además $W \cap V=\emptyset$, pero $x$ se eligió de tal manera que $x \in c l_{X} V$, o sea $W \cap V \neq \emptyset$ lo cual es una contradicción. Se sigue que $\mathcal{F}_{n}$ es un filtro abierto libre en $X$.

(vi) Ahora demostraremos que los filtros $\mathcal{F}_{n}$ y $\mathcal{F}_{m}$, con $n<m$, son Hausdorff separados. Con este fin sea $B \in \mathcal{B}_{n}$; dado que $\mathcal{B}_{m}$ es una familia localmente finita, existe $W_{B}$ abierto en $X$ tal que $p_{B} \in W_{B} \subset B$ y $\mathcal{F}=\left\{C \in \mathcal{B}_{m}: W_{B} \cap C \neq \emptyset\right\}$ es una subfamilia finita de $\mathcal{B}_{m}$. Como $\mathcal{B}_{n} \cap \mathcal{B}_{m}=\emptyset$ si $n \neq m$ entonces $B \neq C$ para todo $C \in \mathcal{F}$, así que $p_{B} \notin\left\{p_{C}: C \in \mathcal{F}\right\}$. Puesto que $X$ es de Hausdorff y $\left\{p_{C}: C \in \mathcal{F}\right\}$ es finito, existe una vecindad abierta $S_{B}$ de $p_{B}$ tal que $\left\{p_{C}: C \in\right.$ $\mathcal{F}\} \cap \operatorname{cl}\left(S_{B}\right)=\emptyset$ y por lo tanto $\left\{p_{C}: C \in \mathcal{F}\right\} \cap \operatorname{cl}\left(W_{B} \cap S_{B}\right)=\emptyset$. Se sigue que $U=\cup\left\{W_{B} \cap S_{B}: B \in \mathcal{B}_{n}\right\} \in \mathcal{F}_{n}$. Por otro lado $c l U \cap\left\{p_{C}: C \in \mathcal{B}_{m}\right\}=\emptyset$, ya que por definición de $U$ y por el hecho de que $\left\{W_{B} \cap S_{B}: B \in \mathcal{B}_{n}\right\}$ es una familia localmente finita se tiene que; $c l U \cap\left\{p_{B}: B \in \mathcal{B}_{m}\right\}=$

$$
\begin{aligned}
& =\cup\left\{c l\left(W_{B} \cap S_{B}\right): B \in \mathcal{B}_{n}\right\} \cap\left\{p_{C}: C \in \mathcal{B}_{m}\right\} \\
& =\cup\left\{c l\left(W_{B} \cap S_{B}\right) \cap\left\{p_{C}: C \in \mathcal{B}_{m}\right\}: B \in \mathcal{B}_{n}\right\} \subset \\
& \cup\left\{c l\left(W_{B} \cap S_{B}\right) \cap\left\{p_{C}: C \in \mathcal{F}\right\}: B \in \mathcal{B}_{n}\right\}=\emptyset
\end{aligned}
$$

Por lo tanto $X \backslash c l U \in \mathcal{F}_{m}$ y se sigue que $\mathcal{F}_{n}$ y $\mathcal{F}_{m}$ son Hausdorff separados.

(vii) Sea $C \in \mathcal{C}(X)$; entonces para todo $n \in \omega$ y para todo $U \in \mathcal{F}_{n}$ se tiene que $U \cap C \neq \emptyset$, si y sólo si $\left\{B \in \mathcal{B}_{n}: p_{B} \in C\right\}$ es infinito. En efecto, si existe $U \in \mathcal{F}_{n}$ tal que $U \cap C=\emptyset$, entonces $\left\{B \in \mathcal{B}_{n}: p_{B} \in C\right\}$ es finito. Inversamente, si $\left\{B \in \mathcal{B}_{n}: p_{B} \in C\right\}$ es finito, entonces $X \backslash C \in \mathcal{F}_{n}$ o sea existe $U \in \mathcal{F}_{n}$ tal que $U \cap C=\emptyset$.

Sea $\mathcal{A}=\left\{C \in \mathcal{C}(X)\right.$ : para todo $n \in \omega$ existe $U \in \mathcal{F}_{n}$ tal que $C \cap U=$ $\emptyset\}$. Si $C \in \mathcal{A}$ entonces $\{B \in \mathcal{B}: B \subset C\}$ es numerable, pues para cada $n,\left\{B \in \mathcal{B}_{n}: p_{B} \in C\right\}$ es finito lo cual implica que $\left\{B \in \mathcal{B}_{n}: B \in C\right\}$ 
es finito. Se sigue que $\left\{B \in \mathcal{B}=\cup \mathcal{B}_{n}: B \subset C\right\}$ es numerable y puesto que $\mathcal{B}$ es una $\pi$-base de $C, \pi w(C) \leq w$.

Sea $\mathcal{M}$ una familia maximal de elementos de $\mathcal{A}$ ajenos dos a dos. Para $n \in \omega$ y $B \in \mathcal{B}_{n}$, sea $\mathcal{A}_{B}=\{C$ : es un subconjunto abierto-cerrado de $X$, tal que $B \subset C \subset M$ para algún $M \in \mathcal{M}\}$, se sigue que $\mathcal{A}_{B}=\emptyset$ si para cada $M \in \mathcal{M}, B \not \subset M$. Ahora, por el Lema 2.15 se tiene que $\left|\mathcal{A}_{B}\right| \leq \mathfrak{c}$, pues $B$ estácontenido en a lo más un elemento de $\mathcal{M} \mathrm{y}$ $\pi w(M) \leq \omega$ para todo $M \in \mathcal{M}$. Ahora definimos por inducción:

$$
\mathcal{M}_{1}=\emptyset \text { y } \quad \mathcal{M}_{n}=\cup\left\{\mathcal{A}_{B}: B \in \mathcal{B}_{n}\right\} \backslash \cup\left\{\mathcal{M}_{k}: k<n\right\} .
$$

Entonces $\left|\mathcal{M}_{n}\right| \leq\left|\cup\left\{\mathcal{A}_{B}: B \in \mathcal{B}_{n}\right\}\right| \leq\left|\mathcal{B}_{n}\right| \mathfrak{c}$ y además afirmamos que $\cup\left\{\mathcal{M}_{n}: n \in \omega\right\}=\{C: C$ es un subconjunto abierto-cerrado no vacío de $X$, tal que $C \subset M$ para algún $M \in \mathcal{M}\}$. Para demostrar la última afirmación consideramos un subconjunto abierto-cerrado no vacío $C \subset X$, tal que $C \subset M$ para algún $M \in \mathcal{M}$; entonces, dado que $\mathcal{B}$ es una $\pi$-base de $X$, existe $B \in \mathcal{B}$ tal que $B \subset C$. Así que, $C \in \mathcal{A}_{B}$ y por lo tanto existe $n \in \omega$ tal que $C \in \mathcal{M}_{n}$, lo cual implica que $\{C: C$ es un subconjunto abierto-cerrado no vacío de $X$, tal que $C \subset M$ para algún $M \in \mathcal{M}\} \subset \cup\left\{\mathcal{M}_{n}: n \in \omega\right\}$. La contención inversa es inmediata.

(viii) Para $C \in \cup\left\{\mathcal{M}_{n}: n \in \omega\right\}$, sea $\gamma_{C}$ un ultrafiltro abierto libre en $X$ tal que $C \in \gamma_{C}$. Se observa que $X \backslash C \in \mathcal{F}_{n}$, ya que, $C \in \cup\left\{\mathcal{M}_{n}: n \in \omega\right\}$, implica que existen $n \in \omega$ y $B \in \mathcal{B}_{n}$ tales que $C \in \mathcal{A}_{B}$ y por lo tanto $C \subset M$ para algún $M \in \mathcal{M}$. Ahora $\mathcal{M} \subset \mathcal{A}$ y por lo tanto, para todo $n \in \omega$ existe $U \in \mathcal{F}_{n}$ tal que $C \cap U=\emptyset$. Por (vii) $\left\{B \in \mathcal{B}_{n}: p_{B} \in C\right\}$ es finito, es decir $\left\{B \in \mathcal{B}_{n}: p_{B} \notin(X \backslash C)\right\}$ es finito, se sigue que $X \backslash C \in \mathcal{F}_{n}$. Por hipótesis $C \in \gamma_{C}$ y como consecuencia, $\gamma_{C}$ y $\mathcal{F}_{n}$ son Hausdorff separados.

(ix) Sea $n \in \omega$, fijo. Definimos,

$U N I F\left(\mathcal{B}_{n}\right)=\left\{\mathcal{U}: \mathcal{U}\right.$ es un ultrafiltro uniforme libre con $\left.\mathcal{B}_{n} \in \mathcal{U}\right\}$ y

$$
\mathcal{G}_{\mathcal{U}}=\left\{U \in T(X):\left\{B \in \mathcal{B}_{n}: q_{B} \in U\right\} \in \mathcal{U}\right\} \text { para } \mathcal{U} \in U N I F\left(\mathcal{B}_{n}\right) \text {. }
$$

Se observa que $\mathcal{G}_{\mathcal{U}}$ es un filtro abierto en $X$, ya que $\emptyset \notin \mathcal{G}_{\mathcal{U}}$, pues $\emptyset \notin \mathcal{U}$. Si $U, V \in \mathcal{G}_{\mathcal{U}}$ entonces $\left\{B \in \mathcal{B}_{n}: q_{B} \in U\right\}$ y $\left\{B \in \mathcal{B}_{n}: q_{B} \in V\right\} \in \mathcal{U}$, 
así que $\left\{B \in \mathcal{B}_{n}: q_{B} \in U \cap V\right\} \in \mathcal{U}$ y por lo tanto se sigue que $U \cap V \in \mathcal{G}_{\mathcal{U}}$. Si $U \in \mathcal{G}_{\mathcal{U}}$ y $V$ es un abierto en $X$ tal que $U \subset V$, entonces $\left\{B \in \mathcal{B}_{n}: q_{B} \in U\right\} \in \mathcal{U}$ y $\left\{B \in \mathcal{B}_{n}: q_{B} \in U\right\} \subset\left\{B \in \mathcal{B}_{n}: q_{B} \in V\right\}$, por lo tanto $\left\{B \in \mathcal{B}_{n}: q_{B} \in V\right\} \in \mathcal{U}$ y se sigue que $V \in \mathcal{G}_{\mathcal{U}}$.

Se afirma que $\mathcal{G}_{\mathcal{U}}$ es un filtro abierto libre, pues si $x \in \cap\left\{c_{X}(U): U \in\right.$ $\left.\mathcal{G}_{\mathcal{U}}\right\}$ y $\mathcal{W} \in \mathcal{U}$, entonces $x \in c l(\cup\{B: B \in \mathcal{W}\})$. Pero $\mathcal{B}_{n}$ es localmente finita, lo cual implica que $x \in \operatorname{cl}(B)$ unicamente para un número finito de $B \in \mathcal{W}$, digamos $x \notin c l(B)$ para cada $B \in \mathcal{W} \backslash \mathcal{F}$, donde $\mathcal{F}$ es finito. Esto es una contradicción, pues $\mathcal{W} \backslash \mathcal{F} \in \mathcal{U}$.

Se puede demostrar, usando argumentos muy parecidos a los que se usaron en (vi), que para todo $n, m \in \omega$ y $\mathcal{U} \in \operatorname{UNIF}\left(\mathcal{B}_{n}\right), \mathcal{V} \in$ $\operatorname{UNIF}\left(\mathcal{B}_{m}\right)$ son ciertos los siguientes incisos:

a) $\mathcal{G}_{\mathfrak{U}}$ y $\mathcal{F}_{m}$ son Hausdorff separados (en el caso de que $m=n$, la prucba usará el hecho de que $p_{B} \neq q_{B}$ para $B \in \mathcal{B}_{n}$ ).

b) Para $n \neq m, \mathcal{G}_{\mathcal{U}}$ y $\mathcal{G}_{\mathcal{V}}$ son Hausdorff separados (la justificación es similar a la dada para demostrar que $\mathcal{F}_{n}$ y $\mathcal{F}_{m}$ son Hausdorff separados y observando que: $\mathcal{B}_{n} \cap \mathcal{B}_{m}=\emptyset$ y $q_{B} \neq q_{C}$ para $B, C \in \mathcal{B}$ y $B \neq C$ ).

c) Para $n-m$ y $\mathcal{U} \neq \mathcal{V}, \mathcal{G}_{\mathcal{U}}$ y $\mathcal{G}_{\mathcal{V}}$ son Hausdorff separados (se usa el hecho de que si $\mathcal{U}, \mathcal{V}$ son ultrafiltros distintos $\operatorname{con} \mathcal{B}_{n} \in \mathcal{U}$ y $\mathcal{B}_{n} \in \mathcal{V}$ entonces $\mathcal{U}$ y $\mathcal{V}$ tienen elementos ajenos).

(x) Sea $M \in \mathcal{M}, C \in C(M)$ y $n \in \omega$. Por (vii) se sigue que, $\mid\{B \in$ $\left.\mathcal{B}_{n}: B \cap C \neq \emptyset\right\} \mid \leq \omega$, por lo tanto, si $\mathcal{U} \in \operatorname{UNIF}\left(\mathcal{B}_{n}\right)$ entonces $\left\{B \in \mathcal{B}_{n}: B \cap C \neq \emptyset\right\} \notin \mathcal{U}$, se sigue que $\mathcal{B}_{n} \backslash\left\{B \in \mathcal{B}_{n}: B \cap C \neq \emptyset\right\} \in \mathcal{U}$, así que por lo definición de $\mathcal{G}_{\mathcal{U}}$, se tiene que $U=\cup\left\{B \in \mathcal{B}_{n}: B \cap C=\right.$ $\emptyset\} \in \mathcal{G}$. Además, por la definición de $U$ se tiene que $U \cap C=\emptyset$ y por lo tanto $U \subset X \backslash C$, lo cual implica que $X \backslash C \in \mathcal{G} u$. Dado que $\gamma_{C}$ es un ultrafiltro abierto libre en $X$ tal que $C \in \gamma_{C}$ y como $X \backslash C \in \mathcal{G}_{U}$ se sigue que $\gamma_{C}$ y $\mathcal{G}_{U}$ son Hausdorff separados. Por otro lado puesto que $\gamma_{C}$ y $\mathcal{G}_{\mathcal{U}}$ son libres, también lo es $\gamma_{C} \cap \mathcal{G}_{\mathcal{U}}$. Obsérvese también, que si $D$. es un subconjunto abierto-cerrado no vacío y $\pi w(D) \leq \omega$ entonces $X \backslash D \in \mathcal{G}_{\mathcal{U}}$ (esto es cierto por los mismos argumentos que prueban que $\left.X \backslash C \in \mathcal{G}_{\mathcal{U}}\right)$. 
Es una consecuencia de $[4$, Teorema 7.8$]$ que,

$$
\left|U^{\prime} N I F\left(\mathcal{B}_{n}\right)\right|=2^{2^{\left|\mathcal{B}_{n}\right|}},
$$

así que (vii) implica que

$$
\left|\mathcal{M}_{n}\right| \leq\left|\cup\left\{\mathcal{A}_{B}: B \in \mathcal{B}_{n}\right\}\right| \leq\left|\mathcal{B}_{n}\right| \mathfrak{c} \leq 2^{2^{\left|B_{n}\right|}}=\left|U N I F\left(\mathcal{B}_{n}\right)\right|
$$

Por lo tanto es posible definir una función inyectiva, $\mathcal{M}_{n} \rightarrow U N I F\left(\mathcal{B}_{n}\right)$. Sea $\mathcal{U}_{C} \in U N \operatorname{IF}\left(\mathcal{B}_{n}\right)$ la imagen de $C \in \mathcal{M}_{n}$. Por (ix), al elemento $\mathcal{U}_{C}$ se le puede asociar un filtro que denotaremos por $\mathcal{G}_{C},\left(\mathcal{G}_{C}=\{U \in\right.$ $\left.\left.T(X):\left\{B \in \mathcal{B}_{n}: q_{B} \in U\right\} \in \mathcal{U}_{C}\right\}\right)$.

(xi) Sea $C, D \in \cup\left\{\mathcal{M}_{n}: n \in \omega\right\}$ tal que $\gamma_{C} \neq \gamma_{D}$, por $(\mathrm{x}), \gamma_{C}$ y $\mathcal{G}_{D}$ son Hausdorff separados y por (ix) (b) y (c), $\mathcal{G}_{C}$ y $\mathcal{G}_{D}$ son Hausdorff separados. Por lo tanto existen, $C_{1}, C_{2} \in \gamma_{C}$ y $D_{1}, D_{2} \in \gamma_{D}$ y $A_{1}, A_{2}, A_{3} \in \mathcal{G}_{C}$ y $B_{1}, B_{2}, B_{3} \in \mathcal{G}_{D}$ tales que $A_{1} \cap C_{1}=\emptyset, A_{2} \cap D_{1}=\emptyset$, $A_{3} \cap B_{1}=\emptyset, B_{2} \cap C_{2}=\emptyset$ y $B_{3} \cap D_{2}=\emptyset$. Tomemos $U=C_{1} \cap C_{2} \in \gamma_{C}$, $V=D_{1} \cap D_{2} \in \gamma_{D}, W=A_{1} \cap A_{2} \cap A_{3} \in \mathcal{G}_{C}$ y $R=B_{1} \cap B_{2} \cap B_{3} \in \mathcal{G}_{D}$, entonces $U, V, W$ y $R$ son ajenos dos a dos. Se sigue que $U \cup W \in \gamma_{C} \cap \mathcal{G}_{C}$ y $V \cup R \in \gamma_{D} \cap \mathcal{G}_{D}$ y $(U \cup W) \cap(V \cup R)=\emptyset$, por lo tanto $\gamma_{C} \cap \mathcal{G}_{C}$ y $\gamma_{D} \cap \mathcal{G}_{D}$ son Hausdorff separados.

Para $C, D \in \cup\left\{\mathcal{M}_{n}: n \in \omega\right\}$ puede suceder que $\gamma_{C}=\gamma_{D}$ (en este caso existe un único $M \in \cup\left\{\mathcal{M}_{n}: n \in \omega\right\}$ tal que $\left.C, D \subset M\right)$. Para $C \in \cup\left\{\mathcal{M}_{n}: n \in \omega\right\}$, sea $H(C)=\left\{D \in \cup\left\{\mathcal{M}_{n}: n \in \omega\right\}: \gamma_{C}=\gamma_{D}\right\}$. En particular, para $C, D \in \cup\left\{\mathcal{M}_{n}: n \in \omega\right\}, \gamma_{C}=\gamma_{D}$ si y sólo si $H(C)=H(D)$.

Ahora, sea

$$
\mathcal{E}=\left\{\mathcal{F}_{n}: n \in \omega\right\} \cup\left\{\gamma_{H(C)} \cap \mathcal{G}_{H(C)}: C \in \cup\left\{\mathcal{M}_{n}: n \in \omega\right\}\right\} .
$$

Mostremos que $\mathcal{E}$ satisface el inciso (a) del Lema 2.9. Para $n \in \omega$ y $C \in \cup\left\{\mathcal{M}_{n}: n \in \omega\right\}$, por (ix) (a) $\mathcal{F}_{n}$ y $\mathcal{G}_{H(C)}$ son Hausdorff separados y por (viii), $\mathcal{F}_{n}$ y $\gamma_{H(C)}$ son Hausdorff separados. Se sigue que $\mathcal{F}_{n}$ y $\gamma_{H(C)} \cap \mathcal{G}_{H(C)}$ son Hausdorff separados. Ahora si $n, m \in \omega$ y $n \neq m, \mathcal{F}_{n}$ y $\mathcal{F}_{m}$ son Hausdorff separados por (vi). Si $C, D \in \cup\left\{\mathcal{M}_{n}: n \in \omega\right\}$ y 
$H(C) \neq H(D), \gamma_{H(C)} \cap \mathcal{G}_{H(C)}$ y $\gamma_{H(D)} \cap \mathcal{G}_{H(D)}$ son Hausdorff separados por (xi). Así que, $\mathcal{E}$ satisface 2.9 (a).

Finalmente mostremos que $\mathcal{E}$ satisface el inciso (b) del Lema 2.9. Sea $C \in \mathcal{C}(X)$. Si existen $n, m \in \omega$ tales que $U \cap C \neq \emptyset$ para todo $U \in \mathcal{F}_{n}$ y $V \cap C \neq \emptyset$ para todo $V \in \mathcal{F}_{m}$; entonces por (ii), $U \cap C \neq \emptyset \neq U \cap(X \backslash C)$ para todo $U \in \mathcal{F}_{\max \{n, m\}}$. Por lo tanto se puede asumir que existe $U \in \mathcal{F}_{n}$ tal que $C \cap U=\emptyset$ (o $\left.(X \backslash C) \cap U=\emptyset\right)$ para toda $n \in \omega$. Supóngase que existe $U \in \mathcal{F}_{n}$ tal que $C \cap U=\emptyset$ (el caso $(X \backslash C) \cap U=\emptyset$ se trabaja de la misma forma) para toda $n \in \omega$. En particular, existe $M \in \mathcal{M}$ tal que $C \cap M \neq \emptyset$. Por (viii), $C \in \gamma_{H(C \cap M)}$ y por (x), $X \backslash C \in \mathcal{G}_{\mathcal{U}_{H(C \cap M)}}$ y se sigue que $C \cap U \neq \emptyset \neq(X \backslash C) \cap U$ para todo $U \in \gamma_{H(C \cap M)} \cap \mathcal{G}_{\mathcal{U}_{H(C \cap M)}}$. Por lo tanto se satisface 2.9 (b).

Se sigue del Lema 2.9 que $X$ tiene una conectificación de Hausdorff.

\section{Definiciones:}

(a) Una función $f: X \rightarrow Y$ es perfecta, si $f$ es cerrada, continua $y f^{-1}[y]$ es compacto para todo $y \in Y$.

(b) La función $f: X \rightarrow Y$ es irreducible, si para todo $A \subset X$ cerrado tal que $f[A]=Y$ entonces $A=X$.

Lema 2.17. Sea $f: X \rightarrow Y$, una función continua, sobreyectiva, cerrada $e$ irreducible. Si $U$ es un subconjunto abierto-cerrado no trivial de $X$, entonces $f[U]$ es un cerrado regular.

Demostración: Si $A \subset X$ sea $f^{\#}(A)=Y \backslash f(X \backslash A)$. Es claro que $f^{\#}(A)=$ $\left\{y \in Y: f^{-1}(y) \subset A\right\}$. Además el ser $f$ irreducible equivale a que $f^{\#}(V) \neq \emptyset$ para cualquier abierto $V \subset X, V \neq \emptyset$. Hagamos $W=f^{\#}(U)$. Como $f(X \backslash U)$ es cerrado en $Y$ el conjunto $W=Y \backslash f(X \backslash U)$ es abierto. Probemos que $c_{Y} W=f(U)$. En efecto, si no es así, entonces existe un $H$ abierto en $Y$ tal que $H \cap f(U) \neq \emptyset$ y $W \cap H=\emptyset$. El conjunto $V=f^{-1}(H) \cap U$ es abierto, no vacío y $f^{\#}(V) \cap W=\emptyset$ ya que esta intersección es subconjunto de $f(V) \cap W \subset H \cap W=\emptyset$. Pero $f^{\#}(V) \neq \emptyset$ y $f^{\#}(V) \subset f^{\#}(U)=W$ lo cual es contradictorio. Entonces $W$ es denso en $f(U)$ y $f(U)$ es cerrado regular. 
La parte (1) del siguiente corolario es parecida al Corolario 2.5, donde como hipótesis, se tiene un espacio metrizable que no es localmente compacto en ningún punto. En el presente corolario se requiere solo que el espacio no tenga subconjuntos abiertos compactos.

\section{Corolario 2.18 .}

(1) todo espacio métrico sin subconjuntos abiertos compactos es conectificable.

(2) Toda preimagen irreducible y perfecta de un espacio métrico el cual no es localmente compacto en ningún punto, tiene una conectificación.

Demostración: (1) Sea $X$ un espacio métrico sin subconjuntos abiertos compactos. Hay dos casos;

(a) Si $\pi w(X)<\omega_{1}$, entonces por ser $X$ un espacio métrico se tiene que $d(X)=\pi w(X)<\omega_{1}$. Además, $X$ no tiene subconjuntos distintos del vacío que sean abierto-cerrados débilmente compactos, pues si fuera así estos subconjuntos serían compactos (por ser $X$ un espacio métrico), es decir $X$ tendría subconjuntos abiertos compactos lo cual es una contradicción. Por el Corolario 2.12, $X$ es conectificable.

(b) $\pi w(X) \geq \omega_{1}$. Dado que $X$ es un espacio métrico, $X$ tiene una $\pi$ base $\sigma$-localmente finita. Además $X$ no tiene subconjuntos abiertos $H$ cerrados distintos del vacío, pues de lo contrario, por ser $X$ un espacio métrico, $X$ tendría subconjuntos abiertos compactos lo cual es una contradicción. Por el Teorema 2.16, $X$ es conectificable.

(2) Sea $Y$ un espacio métrico que no es localmente compacto en ningún punto y sea $f: X \rightarrow Y$ una función perfecta irreducible y sobreyectiva. Dado que $Y$ es métrico, tiene una $\pi$-base $\mathcal{B} \sigma$-localmente finita. Se afirma que $\mathcal{B}^{*}=\left\{f^{-1}[B]: B \in \mathcal{B}\right\}$ es una $\pi$-base $\sigma$-localmente finita para $X$. Con el fin de demostrarlo, sea $U$ un abierto en $X$. Entonces $X \backslash U$ es cerrado en $X$ y como $f$ es una función cerrada se tiene que $f[X \backslash U]$ es cerrado en $Y$, así que $Y \backslash f[X \backslash U]$ es abierto en $Y$, por lo tanto existe $B \in \mathcal{B}$ tal que $B \subset Y \backslash f[X \backslash U]$. Ahora si $x \in f^{-1}[B]$, entonces $f(x) \in B$ y por lo tanto $f(x) \in Y \backslash f[X \backslash U]$, es decir $f(x) \notin f[X \backslash U]$. Asi que $x \notin X \backslash U$, lo cual implica que $x \in U$. Se sigue que $f^{-1}[B] \subset U$. De lo anterior se sigue que $\mathcal{B}^{*}$ es una $\pi$-base para $X$. 
Observemos que si $\mathcal{B}=\cup\left\{\mathcal{B}_{n}: n \in \omega\right\}$ donde $\mathcal{B}_{n}$ es localmente finita para cada $n \in \omega$, entonces $\mathcal{B}^{*}=\cup\left\{\mathcal{B}_{n}^{*}: n \in \omega\right\}$ donde $\mathcal{B}_{n}^{*}=\left\{f^{-1}[B]: B \in \mathcal{B}_{n}\right\}$ para cada $n \in \omega$. Veamos que $\mathcal{B}_{n}^{*}$ es localmente finita para cada $n \in \omega$. Sea $x \in X$, entonces $f(x) \in Y$, por lo tanto existe un abierto $U$ en $Y$ tal que $\left|\left\{B \in \mathcal{B}_{n}: U \cap B \neq \emptyset\right\}\right|<\omega$. Así, dado que $f$ es una función continua se tiene que $f^{-1}[U]$ es un abierto en $X$ que contiene a $x$ tal que $\left|\left\{B \in \mathcal{B}_{n}: f^{-1}[U] \cap f^{-1}[B] \neq \emptyset\right\}\right|<\omega$. Se sigue que $\mathcal{B}_{n}^{*}$ es localmente finita, para cada $n \in \omega$.

De lo anterior se concluye que $X$ tiene una $\pi$-base $\sigma$-localmente finita.

Veamos que $X$ no tiene subconjuntos abiertos $H$-cerrados no vacíos. Si $A$ es un subconjunto abierto-cerrado de $X$, entonces por el Lema $2.17 f[A]$ es un cerrado regular de $Y$ y además dado que $Y$ no es localmente compacto en ningún punto, se sigue que $f[A]$ no es compacto. Ahora como $Y$ es métrico y $f[A]$ no es compacto, se sigue que $f[A]$ no es $H$-cerrado. Como $f\}_{A}: A \rightarrow f[A]$ es una función continua se sigue que $A$ no es $H$-cerrado.

Finalmente ocurren dos casos:

(a) Si $\pi w(X)<\omega_{1}$, entonces $d(X)=\pi w(X)<\omega_{1}$. Además, $X$ no tiene subconjuntos abierto-cerrados distintos del vacío que sean débilmente compactos, pues si existe tal conjunto $A \neq \emptyset$ entonces, dado que $f$ es continua, se sigue que $f[A]$ es débilmente compacto. Pero $Y$ es métrico, por lo tanto $f[A]$ es regular cerrado y compacto lo cual es una contradicción, pues $Y$ no es localmente compacto. Por el Corolario $2.12, X$ es conectificable.

(b) Si $\pi w(X) \geq \omega_{1}$, entonces dado que $X$ tiene una $\pi$-base $\sigma$-localmente finita y además $X$ no tiene subconjuntos abiertos $H$-cerrados distintos del vacío, se sigue del Teorema 2.16 , que $X$ es conectificable.

En [17], se trabaja con espacios de Tychonoff y se establecen condiciones suficientes para que dichos espacios tengan una conectificación de Hausdorff.

Definiciones: Sea $X$ un espacio de Tychonoff. 
(a) Definimos

$$
\begin{gathered}
C(X)=\{f: X \rightarrow \mathbb{R}: f \text { es una función continua }\} \\
\text { y } C^{*}(X)=\{f \in C(X): f \text { es acotada }\}
\end{gathered}
$$

(b) Sea $\mathcal{M}$ un ideal maximal en $C(X)$, (respectivamente $C^{*}(X)$ ). Si la copia canónica de $\mathbb{R}$ en el anillo cociente $C(X) / \mathcal{M}$ es todo el anillo, diremos que $\mathcal{M}$ es un ideal real (si este no es el caso diremos que $\mathcal{M}$ es un ideal hiper-real).

(c) Sea $v X$ el conjunto de puntos $p \in \beta X$ que satisfacen $\{f \in C(X): p \in$ $\left.c l_{\beta X} f^{-1}[0]\right\}$ es real. A $v X$ se le conoce como la realcompactación de Hewitt.

(d) $X$ es n-realcompacto (nearly realcompact) si $\beta X \backslash v X$ es denso en $\beta X \backslash X$.

(e) $A \subset X$, es relativamente pseudocompacto en $X$ si todo $f \in C(X)$ es acotado en $A$.

(f) Un subespacio $Y$ de $X$ está $C$-encajado en $X$ si cada $f \in C(Y)$ puede ser extendida a un elemento $\bar{f} \in C(X)$.

Se observa que la realcompactación de Hewitt de un espacio de Tychonoff $X$ se caracteriza como;

$v X=\{p \in \beta X:$ no existe $f \in C(\beta X)$ tal que $f[X] \subset(0, \infty)$ y $f(p)=0\}$

La siguiente proposición, que aparece en [3], demuestra caracterizaciones para que subconjuntos de un espacio de Tychonoff sean relativamente pseudocompacto.

Proposición 2.19. Si $X$ es un espacio de Tychonoff y $A \subset X$, entonces los siguientes incisos son equivalentes.

(a) A es relativamente pseudocompacto en $X$.

(b) Para toda familia discreta $\left\{U_{n}: n \in \omega\right\}$ de subconjuntos abiertos de $X, U_{n} \cap A=\emptyset$ para algún $n \in \omega$.

(c) $\operatorname{cl}_{\beta X} A \subset v X$. 
(d) $c l_{v X} A$ es compacto.

Demostración: Veamos que (a) implica (b). Supóngase que (b) no es verdadero, entonces existe una familia discreta $\left\{U_{n}: n \in \omega\right\}$ de subconjuntos abiertos de $X$ tales que $U_{n} \cap A \neq \emptyset$ para todo $n \in \omega$, así que existe $x_{n} \in U_{n} \cap A$ para cada $n \in \omega$. Sea $B=\left\{x_{n}: n \in \omega\right\}$, este subconjunto de $A$ es infinito y además discreto pues, por ser $\left\{U_{n}: n \in \omega\right\}$ una familia discreta $U_{n} \cap\left\{x_{n}\right\}=\left\{x_{n}\right\}$ para toda $n \in \omega$. Por el Lema 1.1 de [9], se sigue que $B$ está $C$-encajado en $X$, sea $f: B \rightarrow \mathbb{R}$ una función definida por $f\left(x_{n}\right)=n$ para toda $n \in \omega$, esta función se extiende continuamente a $X$ pero no es acotada en $A$; así que, $A$ no es relativamente pseudocompacto lo cual es una contradicción.

Veamos que (b) implica (c). Supóngase que (c) no es verdadero, entonces existe $p \in c_{\beta X} A \backslash v X$ y así que existe $f \in C(\beta X)$ tal que $f(p)=0$ y $f(q)>0$ para todo $q \in v X$. Sea $g=1 /(f \mid X)$; esta función no es acotada en $A$, pues $f(p)=0$ y por lo tanto existe $\left\{x_{n}: n \in \omega\right\} \subset A$ tal que $1 \leq g\left(x_{n+1}\right)-g\left(x_{n}\right)$ para toda $n \in \omega$. Para cada $n \in \omega$, definimos

$$
U_{n}=g^{-1}\left(g\left(x_{n}\right)-1 / 3, g\left(x_{n}\right)+1 / 3\right) .
$$

Entonces $\left\{U_{n}: n \in \omega\right\}$ es una familia discreta de subconjuntos abiertos de $X$ tales que $U_{n} \cap A \neq \emptyset$, lo cual es una contradicción.

(c) implica (d), pues $c l_{v X} A=c l_{B X} A \cap v X=c l_{B X} A$, el cual es compacto.

Finalmente (d) implica (a), pues si $c_{v X} A$ es compacto y $f: X \rightarrow \mathbb{R}$ es continua, entonces $f$ tiene una extensión continua a $v X$. Por lo tanto $f \mid c l_{v X} A$ es acotada y se sigue que $f \mid A$ es acotado.

Proposición 2.20. Sea $X$ un espacio n-realcompacto. Si $A$ es abiertocerrado y relativamente pseudocompacto en $X$, entonces $A$ es compacto.

Demostración: Dado que $X$ es un espacio $n$-realcompacto y $A$ es un subconjunto abierto-cerrado de $X$, se sigue que $\beta A=c l_{\beta X} A$ y por lo tanto $A$ es $n$-realcompacto, es decir $\beta A \backslash v A$ es denso en $\beta A \backslash A$. Además $A$ es pseudocompacto, por lo tanto $\beta A=v A$. Se sigue que $\emptyset=\beta A \backslash v A$ es denso en $\beta A \backslash A$, así que $\beta A=A$, por lo tanto $A$ es compacto. 
Lema 2.21. Si $A$ es abierto-cerrado y no relativamente pseudocompacto en $X$, entonces $2^{\mathfrak{C}} \leq\left|c l_{\beta X} A \backslash X\right|$.

Demostración: Como $A$ es un subconjunto de $X$ abierto-cerrado y no relativamente pseudocompacto, entonces por la Proposición 2.19 existen $p \in$ $c l_{\beta X} A \backslash v X$ y un conjunto nulo $Z \subset \beta X$, tales que $p \in Z$ y $Z \cap X=\emptyset$. Puesto que $c l_{\beta X} A$ es abierto-cerrado en $\beta X, Z \cap c_{\beta X} A$ es un conjunto nulo de $\beta X$ que no intersecta a $X$. Por $\left[10\right.$, Teorema 9.5] se tiene que, $2^{\mathfrak{c}} \leq\left|Z \cap c_{\beta X} A\right|$ por lo tanto $2^{\mathfrak{C}} \leq\left|c l_{\beta X} A \backslash X\right|$.

La técnica que se usa en la siguiente proposición, para lograr la conectificación de un espacio de Tychonoff es, agregar puntos al espacio y definir un sistema de vecindades en términos de ciertos vecindades en la compactificación de Stone-Čech. Este resultado aparece en [17].

Proposición 2.22. Si $X$ es un espacio de Tychonoff que no tiene más de $2^{\mathfrak{C}}$ subconjuntos abierto-cerrados, ninguno de los cuales es relativamente pseudocompacto, entonces $X$ tiene una conectificación de Hausdorff.

Demostración: Sea $\left\{U_{\alpha}: \alpha<\kappa\right\}$ la colección de todos los subconjuntos abierto-cerrados de $X$ donde $\kappa \leq 2^{\mathfrak{c}}$. Como ningún subconjunto abiertocerrado es relativamente pseudocompacto, por el Lema 2.21, se sigue que $2^{\mathfrak{c}} \leq\left|c l_{3 X} U_{\alpha} \backslash X\right|$ para toda $\alpha<\kappa$. Así que existen puntos distintos $p_{0}$, $q_{0} \in \beta X \backslash X$ tales que $p_{0} \in c l_{\beta X} U_{0} \backslash X$ y $q_{0} \in c l_{\beta X}\left(X \backslash U_{0}\right) \backslash X$. Supóngase que para $\lambda<\alpha$, se tiene la colección;

$$
\left\{p_{\lambda}, q_{\lambda}: p_{\lambda} \in c l_{\beta X} U_{\lambda} \backslash X, \quad q_{\lambda} \in c l_{\beta X}\left(X \backslash U_{\lambda}\right) \backslash X \quad \text { y } \quad \lambda<\alpha\right\},
$$

el cual consiste de puntos de $\beta X \backslash X$, distintos entre si. Como $\lambda<\alpha<\kappa \leq$ $2^{\mathfrak{C}} \leq\left|c l_{\beta X} U_{\alpha} \backslash X\right|$, entonces existirán puntos distintos $p_{\alpha}, q_{\alpha} \in \beta X \backslash X$ tales que $p_{\alpha} \in c_{\beta X} U_{\alpha} \backslash X$ y $q_{\alpha} \in c l_{\beta X}\left(X \backslash U_{\alpha}\right) \backslash X$ y además;

$$
\left\{p_{\alpha}, q_{\alpha}\right\} \cap\left\{p_{\lambda}, q_{\lambda}: p_{\lambda} \in c l_{\beta X} U_{\lambda} \backslash X, \quad q_{\lambda} \in c l_{\beta X}\left(X \backslash U_{\lambda}\right) \backslash X \text { y } \lambda<\alpha\right\}=\emptyset \text {. }
$$

Por lo tanto se puede seleccionar una colección de parejas ajenas $\left\{\left\{p_{\alpha}, q_{\alpha}\right\}\right.$ : $\alpha<\kappa\}$ en $\beta X \backslash X$ con $p_{\alpha} \in c_{\beta X} U_{\alpha} \backslash X$ y $q_{\alpha} \in c_{\beta X}\left(X \backslash U_{\alpha}\right) \backslash X$ y $p_{\alpha} \neq q_{\alpha}$.

Sea $\left\{w_{\alpha}: \alpha<\kappa\right\}$ un conjunto de cardinalidad $\kappa$ tal que $w_{\alpha} \notin X$ para todo $\alpha<\kappa$ y sea $Y=X \cup\left\{w_{\alpha}: \alpha<\kappa\right\}$. Asignamos una topología en $Y$ como sigue: 
(a) $U \subset X$ es abierto en $Y$, si y sólo si, $U$ es abierto en $X$.

(b) Sean $\left\{G_{\alpha}^{i}: i \in I_{\alpha}\right\}$ y $\left\{H_{\alpha}^{j}: j \in J_{\alpha}\right\}$ respectivamente los sistemas de vecindades en $\beta X$ de $p_{\alpha}$ y $q_{\alpha}$. Se define una base local de $w_{\alpha}$ como los conjuntos de la forma;

$$
\left\{\left\{w_{\alpha}\right\} \cup\left(G_{\alpha}^{i} \cap X\right) \cup\left(H_{\alpha}^{j} \cap X\right): i \in I_{\alpha}, j \in J_{\alpha}\right\} .
$$

Veamos que $X$ es denso en $Y$. Sea $U \neq \emptyset$, un subconjunto abierto de $Y$, ocurren dos casos;

(1) si $U \subset X$ entonces $U \cap X \neq \emptyset$.

(2) Si $U \not \subset X$, entonces existe $\alpha<\kappa$ tal que $w_{\alpha} \in U$, por lo tanto existen $i \in I_{\alpha}$ y $j \in J_{\alpha}$ tales que $\left\{w_{\alpha}\right\} \cup\left(G_{\alpha}^{i} \cap X\right) \cup\left(H_{\alpha}^{j} \cap X\right) \subset U$, pero $G_{\alpha}^{i}$ es un abierto en $\beta X$ que contiene a $p_{\alpha}$ y $p_{\alpha} \in c l_{\beta X} U_{\alpha} \backslash X$, así que $\emptyset \neq\left(G_{\alpha}^{i} \cap X\right) \cap U_{\alpha} \subset X$, es $\operatorname{decir} G_{\alpha}^{i} \cap X \neq \emptyset$. Se sigue que $U \cap X \neq \emptyset$. De los dos incisos anteriores se tiene que $X$ es denso en $Y$.

Se tiene que $Y$ es de Hausdorff; en efecto, dados $x \neq y \in Y$ ocurren tres casos;

(1) Si $x, y \in X$ entonces existen abiertos $U, V$ en $X$ (y por lo tanto abiertos en $Y$ ) tales que $x \in U$ y $y \in V$ donde $U \cap V=\emptyset$.

(2) Si $x, y \in Y \backslash X$, entonces existen $\alpha, \lambda<\kappa$ tales que $x=w_{\alpha}, y=w_{\lambda}$. Por construcción $p_{\alpha}, q_{\alpha}$ y $p_{\lambda}, q_{\lambda}$ son puntos distintos de $\beta X$ y dado que $\beta X$ es un espacio de Hausdorff, existen $i \in I_{\alpha}, j \in J_{\alpha}$ y $m \in I_{\lambda}, n \in J_{\lambda}$ tales que $G_{\alpha}^{i}, H_{\alpha}^{i}$ y $G_{\lambda}^{m}, H_{\lambda}^{n}$ son ajenos entre si. Se sigue que;

$$
\left[\left\{w_{\alpha}\right\} \cup\left(G_{\alpha}^{i} \cap X\right) \cup\left(H_{\alpha}^{j} \cap X\right)\right] \cap\left\{\left\{w_{\lambda}\right\} \cup\left(G_{\lambda}^{m} \cap X\right) \cup\left(H_{\lambda}^{n} \cap X\right)\right]=\emptyset .
$$

(3) Si $x \in X$ y $y \in Y \backslash X$, entonces existe $\alpha<\kappa$, tal que $y=w_{\alpha}$. Por construcción $p_{\alpha}$ y $q_{\alpha}$ son puntos distintos de $\beta X \backslash X$ y dado que $x \in \beta X$ y siendo $\beta X$ un espacio de Hausdorff, existen $i \in I_{\alpha}, j \in J_{\alpha}$ y $U$ un abierto en $\beta X$ que contiene a $x$, tales que $G_{\alpha}^{i}$ y $H_{\alpha}^{j}$ son vecindades de $p_{\alpha}$ y $q_{\alpha}$ respectivamente y $U, G_{\alpha}^{i}, H_{\alpha}^{j}$ son ajenos entre si, en consecuencia $U \cap X, G_{\alpha}^{i}, H_{\alpha}^{j}$ son abiertos ajenos en $Y$ que contienen a $x, p_{\alpha}$ y $q_{\alpha}$ respectivamente. Se sigue que $\left[\left\{w_{\alpha}\right\} \cup\left(G_{\alpha}^{i} \cap X\right) \cup\left(H_{\alpha}^{j} \cap X\right)\right] \cap U=\emptyset$. 
De los tres incisos anteriores se concluye que $Y$ es un espacio de Hausdorff.

Por ultimo se afirma que $Y$ es un espacio conexo, pues si no fuera así, entonces existiría $U$ abierto-cerrado no vacío en $Y$ tal que $Y \backslash U \neq \emptyset$. Se sigue que $U \cap X$ y $(Y \backslash U) \cap X=X \backslash(U \cap X)$ son abierto-cerrados no vacíos en $X$. Por construcción existe $\alpha<\kappa$, tal que $U_{\alpha}=U \cap X$ y entonces $p_{\alpha}, q_{\alpha}$, son dos puntos distintos en $\beta X \backslash X$ donde $p_{\alpha} \in c l_{\beta X}[U \cap X]$ y $q_{\alpha} \in$ $c l_{\beta X}[X \backslash(U \cap X)]$. Recordemos que una base local para $w_{\alpha}$ es la familia $\left\{\left\{w_{\alpha}\right\} \cup\left(G_{\alpha}^{i} \cap X\right) \cup\left(H_{\alpha}^{j} \cap X\right): i \in I_{\alpha}, j \in J_{\alpha}\right\}$, donde $G_{\alpha}^{i}$ y $H_{\alpha}^{j}$ son abiertos en $\beta X$ que contienen a $p_{\alpha}$ y a $q_{\alpha}$ respectivamente. Se sigue que $w_{\alpha} \in c l_{\beta X}[U \cap X]$ y $w_{\alpha} \in c l_{\beta X}[X \backslash(U \cap X)]$, lo cual es imposible. Se sigue que $Y$ es conexo.

Lo anterior implica que $Y$ es un espacio de Hausdorff y conexo en el cual $X$ es denso.

Proposición 2.23. Sea $X$ un espacio n-realcompacto, tal que $X$ no tiene subconjuntos abiertos y compactos no vacíos y además que $X$ no tiene más de $2^{\mathfrak{c}}$ subconjuntos abierto-cerrados. Entonces $X$ tiene una conectificación de Hausdorff.

Demostración: Dado que $X$ es un espacio $n$-realcompacto, $X$ no tiene subconjuntos abierto-cerrados relativamente pseudocompactos, pues de ser así estos serían compactos por la Proposición 2.20, pero esto es imposible pues $X$ no tiene subconjuntos abiertos y compactos no vacíos. Ahora $X$ no tiene más de $2^{\mathfrak{c}}$ subconjuntos abierto-cerrados. Se sigue de la Proposición 2.22 que $X$ tiene una conectificacion de Hausdorff.

Se observa que en [20] aparece el Teorema de Weiss, el cual afirma que, si es verdadero el axioma de Martin (AM) y falsa la Hipótesis del Continuo (CH) entonces, todo espacio perfectamente normal y numerablemente compacto es compacto. Con el uso de la observación anterior, se tiene una condición necesaria y suficiente para que un espacio perfectamente normal tenga una conectificación de Hausdorff. 
Proposición 2.24. ( $A M+\neg C H$ ). Si $X$ es un espacio perfectamente normal que no tiene más de $2^{\mathfrak{C}}$ subconjuntos abierto-cerrados, entonces los siguientes incisos son equivalentes:

(1) X tiene una conectificación de Hausdorff.

(2) Ningún subconjunto abierto-cerrado no vacío de $X$ es relativamente pseudocompacto.

Demostración: Veamos que (1) implica (2). Supongamos que $X$ tiene un subconjunto abierto-cerrado $A \neq \emptyset$, tal que $A$ es relativamente pseudocompacto. Puesto que $A$ es abierto-cerrado en $X, A$ es $C$-encajado en $X$ y por lo tanto $A$ es pseudocompacto. Pero $A$ es normal, así que por [7,Teorema 3.9.10] $A$ es numerablemente compacto. Además $X$ es perfectamente normal por lo tanto $A$ también lo es por ser un subconjunto cerrado de $X$. Se sigue del Teorema de Weiss que $A$ es compacto. Por lo tanto $A$ es un subconjunto abierto y compacto de $X$, es decir $X$ no es conectificable lo cual es una contradicción con el inciso (1).

Ahora (2) implica (1) porque se cumplen inmediatamete las hipótesis de la Proposición 2.22, por lo tanto se sigue (1).

\section{Referencias.}

[1] O. T. Alas, M. G. Tkačenko, V. V. Tkachuk y R. G. Wilson, "Connectifying some spaces", Topology and its Applications, 71 (1996) 203-215.

[2] O. T. Alas, M. G. Tkačenko, V. V. Tkachuk, R. G. Wilson y I.V.Yaschenko, "On dense subspaces satisfying stronger separation axioms" Czech. Math. J. Por aparecer.

[3] R. Blair y M. A. Swardson, "Spaces with an Oz Stone- $\check{C}$ ech compactification", Topology and its Aplications, 36 (1990) 73-92.

[4] W. W. Comfort y S. Negrepontis, "The Theory of Ultrafilters". Springer Verlag, New York, 1974.

[5] K. Eda, I. Kamo y T. Nogura, "Spaces which contain a copy of the rationals", J. Math. Soc. Japan, (1990) 103-112. 
[6] A. Emeryk y W. Kulpa, "The Sorgenfrey line has no connected compactification", Comm. Math. Univ. Carolinae, 18 (1977) 483-487.

[7] R. Engelking, "General Topology", PWN Polish Scientific Publishers, Warsaw, 1977.

[8] Z. Frolik, "A generalization of realcompact spaces", Czechoslovak Math. J. 13 (1963) 127-137.

[9] A. García-Maynez y R. G. Wilson, "Classes of topological spaces preserved under realcompactifications". Boletín de la Sociedad Mat. Mex. 31 No. 2, 1986.

[10] L. Gillman, y M. Jerison, "Rings of Continuous Functions", van Nostrand, Princeton, 1960.

[11] V. Neumann-Lara y R. G. Wilson, "When is a LOTS densely orderable?", Topology and its Aplications, 30 (1988) 225-235.

[12] J. R. Porter y R.G. Woods, "Subspaces of connected spaces", Topology and its Aplications, 68 (1996) 113-131.

[13] J. R. Porter y R.G. Woods, "Extensions and Absolutes of Hausdorff Spaces", Springer Verlag, New York, 1987.

[14] J. Porter y J. Vermeer, "Spaces with coarser minimal Hausdorff topologies", Trans. A. M. S. 289, No. 1 (1985), 59-71.

[15] P. Roy, "A countable connected Urysohn space with a dispersion point", Duke, 33 (1966) 331-333.

[16] M. E. Rudin, "Martin's Axiom", in: J. Barwise, ed. Handbook of Mathematical Logic (North-Holland, Amsterdam, 1997) 409-501.

[17] M. A. Swardson, "Nearly realcompact spaces and $T_{2}$-connectifiability". Publicación Preliminar, 1997.

[18] A. Tychonoff, "Über die topologische Erweiterung von Räumen", Math. Ann., 102 (1930) 544-561. 
[19] S. Watson y R. G. Wilson, "Embedding in cornected spaces", Houston J. Math. 19 (1993) 469-481.

[20] W. Weiss, "Countably compact spaces and Martin's Axiom", Can. J. Math. 30 (1978) 243-249. 